\title{
La nanomedicina. Marco normativo regulador y retos jurídicos
}

\section{The nanomedicine. Regulatory framework and legal challenges}

\author{
Juan Alejandro Martínez Navarro ${ }^{1}$ \\ Profesor Sustituto Interino \\ Universidad de Almería
}

SUMARIO: I. INTRODUGGIÓN. II. ASPECTOS GENERALES. UNA APROXIMACIÓN A LA NANOMEDICINA. 1. El desarrollo de la nanociencia. 2. Una aproximación al término «nanomedicina». 3. Clasificación. Las aplicaciones de la nanomedicina. A. La nanomedicina como sistema de diagnóstico. B. La nanomedicina para el suministro de fármacos. C. La nanomedicina regenerativa. III. GRUPO NORMATIVO REGULADOR APLICABLE A LA NANOMEDICINA. 1. La nanomedicina en el ordenamiento jurídico de la Unión Europea. A. La regulación aplicable de los nanomateriales. B. La regulación aplicable a la nanomedicina. C. European Technology Platform Nanomedicine. 2. La nanomedicina en el ordenamiento jurídico español. IV. LOS RETOS JURÍDICOS QUE PLANTEA LA NANOMEDICINA. 1. El desarrollo de un grupo normativo regulador aplicable a la nanomedicina. A. La investigación como elemento esencial para una adecuada regulación. B. La necesidad de un consenso sobre el término «nanomaterial». 2. La nanomedicina: medicamento vs producto sanitario. A propósito del Reglamento (UE) 2017/745. 3. La ética aplicada a la nanociencia. V. A MODO DE GONCLUSIÓN. VI. BIBLIOGRAFÍA.

RESUMEN: La nanotecnología ha llegado para cambiar nuestra sociedad. Su carácter multidisciplinar permitirá rediseñar áreas de estudio como la robótica, la biología y la medicina. En su aplicación con el ámbito de la salud, la nanociencia ha formado una nueva área de investigación, la nanomedicina, con posibilidades y utilidades que modificarán sustancialmente los mecanismos de diagnóstico y trata-

1 Jmn055@ual.es

Recibido: 01/11/2020

Aceptado: 12/11/2020 
miento asistencial: dando un nuevo impulso a la medicina personalizada; desarrollando numerosas herramientas de medicina regenerativa; aprovechando las nuevas propiedades que otorgan las nanopartículas; y un largo etcétera. No obstante, toda nueva tecnología plantea importantes retos. Desde las ciencias jurídicas se deberá afrontar el complejo desafío que supone regular una materia tan novedosa, compleja y amplia como es la nanociencia.

PALABRAS CLAVE: Nanotecnología. Nanomedicina. Ciencias Jurídicas. Ética. Salud.

ABSTRACT: Nanotechnology has come to change our society. Its multidisciplinary nature will allow the redesign of study areas such as robotics, biology and medicine. In its application to the health field, nanoscience has formed a new area of research, nanomedicine, with possibilities and utilities that will substantially modify the mechanisms of diagnosis and care treatment: giving a new impetus to personalized medicine; developing numerous regenerative medicine tools; taking advantage of the new properties that nanoparticles provide; And a long etcetera. However, any new technology poses significant challenges. From the legal sciences, the complex challenge of regulating such a novel, complex and broad subject as nanoscience must be faced.

KEY WORDS: Nanotechnology. Nanomedicine. Legal sciences. Ethics. Health.

\section{INTRODUGGIÓN}

La nanotecnología es identificada como uno de los principales avances científicos de nuestro tiempo. Su trascendencia se extiende a multitud de áreas y campos científico-técnicos, y entre todos destaca el ámbito de la salud.

Las importantes aplicaciones que está generando la nanotecnología se traducirán en importantes beneficios sanitarios y sociales. Al respecto, la tendencia que sigue la evolución de la población europea deriva en importantes retos para los sistemas sanitarios públicos. A corto plazo los sistemas sanitarios públicos europeos tendrán que afrontar el envejecimiento de la población, la medicalización de la sociedad, efectos provocados por los enormes cambios culturales y familiares, atención sanitaria a las personas que llegan de fuera, la dificultad de sostener financieramente el ritmo de la innovación tecnológica de la sanidad moderna, además de los retos que se presentan de forma cíclica derivados de las enfermedades que afectan a la seguridad mundial².

2 PÉREZ GÁLVEZ, J. F., "La sostenibilidad del Sistema Nacional de Salud”, en J. F. PÉREZ GÁLVEZ (dir.), La sostenibilidad del Sistema Nacional de Salud en el siglo XXI, Comares, Granada, 2015, p. 27. 
De modo que, la implementación de los avances de la nanotecnología en el ámbito médico y clínico servirá de inestimable ayuda para afrontar los importantes retos demográficos que se plantean en los países europeos. A largo plazo, permitirá hacer frente al problema del envejecimiento de la población, que está exigiendo una carga cada vez mayor en las redes de atención social y sanitaria. ${ }^{3}$. A modo de ejemplo, la nanomedicina tiene el potencial de permitir la detección temprana y la prevención, así como de mejorar drásticamente el diagnóstico, tratamiento y seguimiento de muchas enfermedades incluyendo el cáncer. Nos encontramos ante un instrumento clave para la medicina personalizada, específica y regenerativa mediante la entrega de nuevos fármacos, tratamientos y dispositivos implantables a médicos y pacientes, por los avances reales en la asistencia sanitaria ${ }^{4}$.

Si bien, las posibilidades que la nanotecnología puede aportar son difíciles de cuantificar. Se prevé que esta tecnología pueda dirigir a la medicina hacia un nuevo y revolucionario rumbo que permita al cuerpo humano repararse a sí mismo. De este modo, los biomateriales inteligentes aplicados en la terapia celular avanzada, permitirán que los propios mecanismos de auto reparación del cuerpo se pueden aprovechar para regenerar o reemplazar tejidos u órganos dañados. Los biomateriales nanoestructurados sirven para iniciar, estimular y dirigir el crecimiento de nuevo tejido en la forma requerida con la función correcta ${ }^{5}$. En la actualidad, existen

3 COMISIÓN EUROPEA, Nanotechnology: the invisible giant tackling Europe's future challenges, Publicaciones Oficina de la Unión Europea, Bélgica, 2013, p. 18. En la actualidad, una de cada cinco personas supera los de 65 años de edad, una proporción que se espera que crezca a uno de cada cuatro en los próximos dos decenios. Las implicaciones son alarmantes: uno de cada tres personas es propensa a desarrollar cáncer, 1,7 millones de muertes relacionadas con el cáncer cada año sólo en Europa. La nanotecnología podría transformar el tratamiento del cáncer en un futuro, así como la de toda una serie de otras condiciones crónicas y debilitantes de la vejez, incluyendo enfermedades cardiovasculares, reumatoide y osteoartritis y enfermedades neurodegenerativas (Alzheimer y Parkinson). El objetivo es la mejora de los resultados de los pacientes, reduciendo a largo plazo los costes de atención social y sanitaria haciendo ésta más accesible.

4 COMISIÓN EUROPEA (2013: 20). Las técnicas como la resonancia magnética (RM), rayos $\mathrm{X}$, tomografía computarizada (TC), y tomografía por emisión de positrones (PET) se utilizan habitualmente para seguir el progreso de la enfermedad y controlar los efectos del tratamiento. En la actualidad, las partículas magnéticas de gadolinio se están empleando como un agente de contraste para mejorar la calidad de la información recogida por resonancia magnética. Tomando este enfoque a nanoescala no sólo podría mejorar la resolución, potencialmente hasta el nivel de una sola célula, sino aportar otras ventajas.

COMISIÓN EUROPEA (2013: 21). 
multitud de proyectos enfocados en la regeneración de tejidos cardíacos ${ }^{6}$, hueso y cartílago ${ }^{7}$ e incluso piel $^{8}{ }^{9}$.

No obstante, el desarrollo de un nuevo campo tan prometedor como es la nanotecnología supone la aparición de importantes y complejos retos. Por ello, es conveniente valorar si las posibilidades que la nanomedicina podría ofrecer en el futuro son contrapeso suficiente frente a los posibles riesgos derivados de esta nueva tecnología. Así, es de suma importancia examinar por adelantado y con extremo cuidado y responsabilidad los posibles desafíos jurídicos que pueda plantear este campo.

\section{ASPEGTOS GENERALES. UNA APROXIMAGIÓN A LA NANO- MEDICINA}

\section{El desarrollo de la nanociencia}

Hace más de una década que asistimos al enorme desarrollo de la nanociencia y la nanotecnología tanto en los países avanzados como en los emergentes ${ }^{10}$. No cabe duda de que nos encontramos ante toda una revolución científica presente en multitud de áreas ${ }^{11}$.

La irrupción de la nanociencia y la nanotecnología y cómo éstas han emergido en la sociedad actual se debe a diversos factores: en primer lugar, al propio avance de la ciencia, que a lo largo de las últimas décadas ha logrado controlar la materia a escalas cada vez más pequeñas, pasando de lo micro a lo nano. En segun-

$6 \mathrm{Al}$ respecto el proyecto NANOCARD financiado por la UE: Vide www.mf.mpg.de/NanoCARD/.

$\mathrm{Al}$ respecto el proyecto OPHIS y INNOVABON financiado por la UE: Vide www.innovabone.eu/.

$8 \mathrm{Al}$ respecto el proyecto ARTIVASC 3D financiado por la UE: Vide www.artivasc.eu/.

$9 \mathrm{Al}$ respecto el proyecto NANOBIOTOUCH financiado por la UE. Disponible en el siguiente enlace: http://nanobiotouch.org/. Una mejor comprensión del funcionamiento físico del cuerpo mediante moléculas a nanoescala, reúne a toda una serie de disciplinas de la ciencia cognitiva desde la ingeniería hasta la biología celular. Se espera el desarrollo de una nueva generación de dispositivos robóticos, que transformará la vida de las personas con discapacidad física o discapacidades visuales. El uso de sensores basados en el sistema de nanoelectromecánicos (NEMS) forma parte de un proyecto respaldado por la UE que se encuentra desarrollando un dedo robótico táctil. No sólo es posible que un sistema de este tipo pueda ayudar a las personas con discapacidad, sino que también podría ser utilizado para la exploración espacial, ambientes extremos o pruebas de productos.

10 SERENA, P. A.; CORREIA, A.; SÁENZ, J. J.; y PÉREZ, M., "Nanotechnology applications: a driving force for R\&D investment", en Physica Staus Solidi, Vol. 204 (2007), pp. 1611-1622. CORREIA, A.; PÉREZ, M.; SÁENZ, J. J.; y SERENA, P. A., "Nanoscience and nanotechnology: driving research and applications", Physica Staus Solidi, Vol. 1 (2007), pp. 68-72.

11 SERENA, P. A.; y CORREIA, A., "Nanotecnología: el motor de la próxima revolución tecnológica”, en Apuntes de Ciencia y Tecnología, núm. 9 (2003), pp. 32-42. 
do lugar, la nanotecnología supone ir un paso más allá en la revolución electrónica actual generando nuevos paradigmas científico-tecnológicos. En tercer lugar, es la consecuencia de mecanismos de producción cada vez más competitivos que permiten a las potencias actuales competir con los países emergentes. Finalmente, se espera que la nanotecnología permita producir nuevos materiales capaces de mejorar a los ya existentes y aportar mejoras a los modelos de financiación, estableciendo modelos económicos más sostenibles y respetuosos con el medioambiente.

Podemos situar el origen de la nanotecnología en el discurso titulado «Hay mucho lugar al fondo» (There is plenty of room at the bottom), pronunciado por el físico y ganador del Premio Nobel Richard Feynman en el año 195912. En su discurso, Feynman expuso la posibilidad de fabricar materiales «átomo por átomo», manipulando la materia a una escala atómica y molecular. Decía Feynman en su discurso: «los principios de la física, tal como yo los veo, no niegan la posibilidad de manipular las cosas átomo por átomo. Al no violar ninguna ley, no hay motivo para que no pueda hacerse [...] En el mundo de lo muy, muy pequeño, muchas cosas nuevas podrán suceder, porque los átomos se comportan de manera distinta a como lo hacen los objetos a mayor escala, pues deben satisfacer las leyes de la mecánica cuántica [...] A nivel atómico, aparecen nuevos tipos de fuerza, nuevas posibilidades, nuevos efectos» ${ }^{13}$. Sin embargo, el término «nanotecnología» fue usado por primera vez por Norio Taniguchi, ingeniero y también ganador del Premio Nobel en $1974^{14}$.

El inicio de la apuesta inversora se originó en los EE.UU. en 1996 cuando varias agencias federales lanzaron la Iniciativa Nacional en Nanotecnología (NNI, National Nanotechnology Initiative $)^{15}$. En lo referente a la Unión Europea, podemos

12 Vide "Plenty of Room at the Bottom", (2001), http://www.its.caltech.edu/ feynman/plenty.html.

13 ODDONE, N.; ZAMBRANA, A.; BERVEJILLO, V.; ALBERRO, A.; y otros, "De la nanobiomoléculas a la nanobiología y nanomedicina", en Revista Interdisciplinaria en Nanociencias y Nanotecnología, Vol. 6, núm. 10 (2013), p. 46.

14 MEJIAS SÁNCHEZ, Y; CABRERA CRUZ, N.; TOLEDO FERNÁNDEZ, A. M.; y DUANY MACHADO, O. J., "La nanotecnología y sus posibilidades de aplicación en el campo científico-tecnológico", en Revista Cubana de Salud Pública, Vol. 35, núm. 3 (2009), p. 3.

15 En la actualidad, Estados Unidos destaca como la principal potencia mundial en el desarrollo y tratamiento de la nanotecnología y sus derivados. La Food and Drug Administration (FDA) es la responsable de «proteger la salud pública mediante la regulación de los medicamentos de uso humano y veterinario, vacunas y otros productos biológicos, dispositivos médicos, el abastecimiento de alimentos en nuestro país, los cosméticos, los suplementos dietéticos y los productos que emiten radiaciones; favorecer la salud pública mediante el fomento de las innovaciones de productos; proveer al público la información necesaria, exacta, con base científica, que le permita utilizar medicamentos y alimentos para mejorar su salud». En cuanto a su organización, la FDA se estructura en varios centros que se especializan en la regulación de determinados tipos de productos. Los que tienen más probabilidades de estar involucrados en la regulación de los productos de la nanomedicina son: el «Centro para la Evaluación e Investigación de Medicamentos» (CDER), el «Centro de Productos Biológicos de Evaluación e Investigación» (CBER), y el «Centro para Dispositivos y 
situar el inicio de la inversión en el VI y VII Programas Marco, a partir de los cuales se adoptaron importantes iniciativas. Entre estas iniciativas cabe destacar la creación de un área temática específica denominada «Nanociencias, Nanotecnologías, Materiales y Nuevas Técnicas de Producción» (NMP) ${ }^{16}$, la creación de Plataformas Tecnológicas como la de Nanoelectrónica (ENIAC) o la de Nanomedicina (NanoMED), así como la asignación de recursos (destinados a la nanotecnología) en otras áreas como el Transporte, la Energía, la Salud, etc ${ }^{17}$.

En la actualidad, tras muchos años de inversión en este sector, nos encontramos aun en una etapa muy primaria de desarrollo atendiendo a las posibilidades que ofrece y al desarrollo que puede alcanzar. Siguiendo a Sancén Contreras, podemos dividir el proceso de desarrollo de la nanotecnología en 4 etapas $^{18}$ : una primera etapa desde los inicios de esta tecnología hasta aproximadamente el año 2000 donde se desarrolla una primera generación de productos que consisten en nanoestructuras pasivas (nanoestructuras dispersas y de contacto, como aerosoles, coloides, etc.). La segunda etapa que se prolonga hasta 2005 se caracteriza por el desarrollo de nanoestructuras activas (durante esta etapa destaca el desarrollo de estructuras bioactivas con efectos sobre la salud, es decir, medicamentos o dispositivos médicos). La tercera etapa hasta el año 2010 se ha caracterizado por el desarrollo de sistemas de nanosistemas, como nuevas arquitecturas jerárquicas, robótica y biosistemas evolutivos. Finalmente, la cuarta etapa se está desarrollando en la actualidad, se espera el desarrollo de nanosistemas moleculares, nanosistemas de diseño atómico y otras funciones emergentes ${ }^{19}$.

Salud Radiológica» (CDRH). A los efectos de la regulación, la FDA clasifica los productos médicos, ya sea como medicamentos, dispositivos, productos biológicos, o su combinación productos. En 2011, la FDA dio un nuevo impulso a su política en materia de nanotecnología publicando unas directrices para ayudar a la industria y los desarrolladores a identificar la seguridad, eficacia o problemas de salud que podrían surgir del uso de determinados nanomateriales.

16 Página oficial disponible en el siguiente enlace: Vide http://www.cordis.lu/nmp/home.html.

17 MORENO GONZÁLEZ, J. y VALENZUELA JUAN, R., "Nanotecnología aplicada a la salud", en Revista I+D, núm. 79 (2010), p. 7. Los Estados miembros también han aportado una importante inversión durante la última década. Investigadores europeos se han asociado con sus similares de los Balcanes occidentales, norte de África y Latinoamérica para fortalecer la cooperación en los campos de la informática biomédica, las tecnologías de malla (grid) y la nanoinformática. Este trabajo se llevó a cabo a través de ACTION-Grid (ACCION-Malla), un proyecto con un presupuesto cercano al millón de euros, financiado por la Unión Europea dentro del VII Programa Marco.

18 SANCÉN CONTRERAS, F., "Nanoética y nanomedicina. Apuntes para una nueva ética de la medicina", en Revista Interdisciplinaria en Nanociencia y Nanotecnología, Vol. 3, núm. 1 (2010), p. 80.

19 POOLE, C. P. y OWENS, F. J., "Introduction to the Nanotechnology", en Wiley-VCH, Weinheim, (2003), p. 20. Por otro lado, Poole y Owens identifican dos fases en la evolución de la nanotecnología. Una primera fase denominada «top-down» (de arriba-abajo), mediante la cual se busca lo pequeño a partir de lo grande, es decir, a partir de elementos complejos se obtienen nanopartículas o moléculas con diferentes usos o aplicaciones. Esta fase está plenamente integrada en nuestra sociedad, sobre todo en sectores 


\section{Una aproximación al término «nanomedicina»}

Según el Instituto Nacional de Tecnología Industrial (INTI) ${ }^{20}$, «la nanotecnología puede definirse como la ingeniería de la materia a escalas de menos de 100 $n m$ con el propósito de obtener propiedades y funciones dependientes del tamaño» ${ }^{21}$. La nanotecnología engloba todas aquellas técnicas y ciencias dedicadas al estudio, diseño, creación, síntesis, control, manipulación y aplicación de materiales, aparatos y sistemas funcionales a una nanoescala, es decir a una millonésima parte de un milímetro $\left(10^{-9}\right.$ metros $)$ y por tanto permiten trabajar y manipular de forma individual átomos y moléculas. No obstante, lo más interesante de la nanotecnología no es la posibilidad de trabajar con materiales de reducidas dimensiones, «sino el cambio a menudo radical que sufren las propiedades físicas y químicas de la materia cuando se trabaja a esta escala: la conductividad eléctrica, el color, la resistencia o la elasticidad, entre otras propiedades, se comportan de manera diferente a como lo hace el material volumétrico» ${ }^{22}$.

De la unión de la nanotecnología y la salud ha surgido toda un área de investigación denominada «nanomedicina» ${ }^{23}$, cuyo objeto principal es desarrollar herra-

como la industria farmacéutica. La segunda fase se denomina «bottom-up» (de abajo-arriba), mediante la cual se busca crear elementos complejos a partir de elementos funcionales o atómicos. Es la gran revolución que ofrece la nanotecnología y supone el dominio absoluto de la nanociencia. No obstante, se estima que la segunda fase no se alcanzará hasta dentro de un par de décadas. De este modo, en muchos sentidos la nanociencia es más un conjunto de expectativas o posibilidades que de realidades funcionales, lo que dificulta enormemente su delimitación.

20 En este sentido, la National Nanotechnology Initative (NNI) define la nanotecnología como el área encargada del estudio, diseño, caracterización, síntesis y aplicación de materiales y sistemas, cuyas estructuras y componentes exhiben nuevas y mejoradas propiedades físicas, químicas o biológicas debido a su tamaño nanomético. CHAN, J. M.; VALENGIA, P. M.; ZHANG, L.; LANGER, R.; y FAROKHZAD, O. C., "Polymeric nanoparticles for drug delivery", en Methods in Molecular Biology, núm. 624 (2010), pp. 163175. ALCALÁ-ALCALÁ, S. y QUINTANAR-GUERRERO, D., "La terapia a nanoescala: ensamblaje de estructuras liberadoras de fármacos", en Revista Interdisciplinaria en Nanociencias y Nanotecnología, Vol. 7, núm. 12 (2014), p. 33.

21 ODDONE, N.; ZAMBRANA, A.; BERVEJILLO, V.; ALBERRO, A.; y otros, "De la nanobiomoléculas a la nanobiología y nanomedicina", en Revista Interdisciplinaria en Nanociencias y Nanotecnología, Vol. 6, núm. 10 (2013), p. 46: «El prefijo "nano" significa la mil millonésima parte de algo. En el caso de un nanómetro, significa la mil millonésima parte de un metro, es decir 10-9m. Dado que muchas de las propiedades físicas y químicas de la materia se modifican en tamaños comprendidos entre 0.1 y 100 nanómetros, éste sería el mundo "nano" estrictamente hablando».

22 LECHUGA, L. M., "Nanomedicina: ampliación de la nanotecnología en la salud", en Curso de Biotecnología aplicada a la salud humana, Grupo de nanobiosensores y aplicaciones bioanalíticas, Consejo Superior de Investigaciones Científicas, Madrid, 2010, p. 98.

23 ECHEVERRÍA-CASTILLO, F., "Retos de este siglo: nanotecnología y salud", en Revista Cubana Hematología, Inmunología y Hemoterapia, núm. 29 (2013), p. 6: «Resulta complicado entender cómo se pueden relacionar estos dos campos si no se tiene una noción de lo que es el mundo nano. Nano (deriva del griego nanno que significa pequeño); no es más que un prefijo que indica una unidad de medida (por 
mientas para diagnosticar, prevenir y tratar enfermedades cuando están todavía en estados poco avanzados o en el inicio de su desarrollo.

Así, la nanomedicina es una rama de la nanotecnología con aplicaciones directas en medicina, que está permitiendo el abordaje de las enfermedades desde el interior del organismo a un nivel celular o molecular ${ }^{24}$. Según la European Technology Platform Nanomedicine (ETPN), la nanomedicina «es la aplicación de la nanotecnología para lograr la innovación en la asistencia sanitaria. Utiliza las propiedades desarrolladas por un material en su escala nanométrica que a menudo difieren en términos de la física, la química o la biología del mismo material en una escala más grande $»^{25}$. Para ello se apoya en dispositivos, sistemas y tecnologías que incluyen nanoestructuras capaces de interactuar a escala molecular.

De un modo similar, desde la doctrina se define la nanomedicina «como uno de los campos de las nanobiotecnologías con aplicaciones directas en medicina, se puede definir como la ciencia y la tecnología utilizada en el diseño y evaluación de sistemas complejos, a escala nanométrica, formados por al menos dos componentes, uno de los cuales es el principio activo o molécula biológicamente activa y, el segundo, es el propio sistema que permite una función especial relacionada con el diagnóstico, tratamiento, o prevención de una enfermedad $»^{26}$.

En definitiva, la nanomedicina es una rama científico-técnica multidisciplinar, que en el ámbito de la salud y junto a las Tecnologías de la Información y la Comunicación supone una aplicación más de la salud electrónica, con posibilidades aun incalculables. Establecer cualquier concreción respecto de la nanotecnología es una tarea compleja, más aún delimitar su naturaleza jurídica. Como principales causas de este motivo destacan la juventud de la nanociencia y su carácter transversal. Si atendemos a las posibilidades que puede aportar la nanotecnología, y a las expectativas creadas, no cabe duda de que se encuentra aún en una fase de crecimiento muy temprana, como dicen algunos expertos, «está en la prehistoria

ejemplo: 1 nanómetro, 10-9 m), aunque en términos de nanociencia y nanotecnología abarca todo aquello que se encuentre desde la escala microscópica hasta la escala nanométrica (10-6 10-9 m); o dicho de otra manera, desde la escala molecular hasta la escala atómica.8 En principio, la nanociencia se dedica al estudio de las propiedades de los objetos y fenómenos a escala nanométrica y a su vez la nanotecnología se ocupa de la manipulación "controlada" y la producción de objetos materiales, instrumentos, estructuras y sistemas a dicha escala.9 Esta es precisamente la escala donde comienza la vida».

24 GUADROS GELORRIO, M.; LLANOS MÉNDEZ, A.; y VILLEGAS PORTERO, R., Nanotecnología en Medicina. Informe de síntesis de tecnología emergente, Agencia de Evaluación de Tecnologías Sanitarias de Andalucía, Sevilla, 2010, p. 20.

25 Vide ETPN, "The potential of nanomedicine: Why is small different", http://www.etp-nanomedicine.eu/public/about-nanomedicine/what-is-nanomedicine.

26 IRACHE, J. M., "Nanomedicina: nanopartículas con aplicaciones médicas", en Anales del Sistema Sanitario en Navarra., Vol. 31, núm. 1 (2008), p. 7. 
desde el punto de vista de su desarrollo» ${ }^{27}$. Supone todo un cambio de paradigma en cuanto a la fabricación y elaboración de bienes y toda una revolución en la búsqueda de elementos funcionales atómicos y moleculares.

\section{Glasificación. Las aplicaciones de la nanomedicina}

«Desde el origen de la revolución industrial, periódicamente (aproximadamente cada 20 años) se ha producido algún avance de enorme importancia en ciencia básica; a continuación, este avance se utilizaba en diferentes aplicaciones, generando nuevas tecnologías y posibilidades, así como riqueza; finalmente, las nuevas tecnologías entraban en una fase de rendimiento decreciente, se convertían en poco rentables (hasta ahora esta última fase coincidía con un nuevo desarrollo). Actualmente nos encontramos en un periodo fascinante, que se caracteriza por la capacidad de controlar la materia en escala atómica. Hoy día sólo vemos las primeras olas del "tsunami" de la nanotecnología: cada vez más productos la contienen. Sin embargo, a lo largo del siglo XXI todo a nuestro alrededor (la ropa, nuestra casa, nuestro coche $[\ldots])$ va a contener elementos de nanotecnología» ${ }^{28}$.

De este modo, a pesar de su corta vida, a través de la nanotecnología se han ramificado importantes áreas de actuación, lo que permite establecer una clasificación de la nanociencia según el área de aplicación, a saber: nanomedicina, nanobiolo-

27 CORREIA, A. y SERENA, P. A., "Introducción", en A. CORRIEA (coord.), Nanociencia y Nanotecnología en España. Un análisis de la situación presente y de las perspectivas de futuro, Phantoms foundation, España, 2011 , p. 11.

28 MIRANDA SORIANO, R., "El tsunami de la nanotecnología", resumen ejecutivo de la sesión impartida el 11 de marzo, Madrid, 2015, p. 2. 
gía ${ }^{29}$, nanoelectrónica ${ }^{30}$, nanoquímica ${ }^{31}$, nanóptica ${ }^{32}$, nanofotónica ${ }^{33}$, nanomateria$\mathrm{les}^{34}$, sondas SPM, energía, nanotubos de carbono ${ }^{35}$, simulación y modelado, nanoin-

29 SAMITIER, J., "Nanobiología y Nanomedicina”, en A. CORREIA (coord.), Nanociencia y Nanotecnología en España. Un análisis de la situación presente y de las perspectivas de futuro, Phantoms foundation, España, 2011, p. 63: «La nanobiotecnología o aplicaciones de la nanotecnología a los sistemas biológicos tiene su área de aportación en medicina mediante nuevos sistemas de diagnóstico tanto a nivel molecular como por técnicas de imagen, nuevas terapias más selectivas y eficientes y como soporte tecnológico a la medicina regenerativa. Así mismo, parte de estos descubrimientos tendrá también influencia en la industria agrícola y de la alimentación».

30 ECHANDI PACHECO, R., "Nanoelectrónica: la nueva era de la miniaturización y la física cuántica”, en Revista Rhombus, Vol. 2, núm. 5 (2006), p. 2: «La nanoelectrónica se refiere a la tecnología de diseño, fabricación y aplicación de dispositivos en una escala de nanómetros, o sea, un billón de metro (10-9 $\mathrm{m}$ ), aproximadamente los diámetros juntos de diez átomos. Con esta nueva especialidad, se puede reducir substancialmente el tamaño de los circuitos integrados e incrementar la velocidad de procesamiento de las computadoras, lo que abre un mundo de nuevas aplicaciones y posibilidades».

31 AGUAYO GONZÁLEZ, F.; ZARZUELA ROLDÁN, E.; y LAMA RUIZ, J. R., "Nanotecnología y nanoquímica”, en Sevilla Técnica, núm. 36 (2011), p. 35: «Bajo el término de Nanoquímica se engloban todas aquellas actividades de la Nanociencia y la Nanotecnología que poseen en común la utilización de las aproximaciones y las herramientas tradicionales de la Química para crear, desarrollar y estudiar objetos que presenten propiedades útiles debido a sus dimensiones nanoscópicas».

32 LOZANO, A., "El nanomagnetismo y la nanóptica centrarán parte de la labor de nanoGUNE", en Diario Tecnología Vasca, 11 de junio de 2011, p. 27. La nanóptica «focaliza su actividad en torno al desarrollo de un nuevo tipo de microscopía óptica para la visualización de nanoestructuras [...] ante la incapacidad de la microscopía óptica tradicional para resolver estructuras inferiores a unos centenares de nanómetros».

33 DE LA ROSA CRUZ, E., "Nanofotónica: luz + nanopartículas", en Ide@s CONCTTEG, 24, núm. 2 (2007), p. 414: «La nanofotónica es la fusión de la nanotecnología y la fotónica. Es un campo multidisciplinario que estudia las propiedades ópticas de los sistemas nanoestructurados y la interacción luz-materia a nivel nanoscópico».

34 ZEA, R., "Nanomaterials: health effects and legislation", en Ingeniería e investigación, Vol. 32, núm. 1 (2012), p. 36. La International Organization for Standardization (ISO) define los nanomateriales como aquellos materiales con dimensiones a nanoescala (desde 1 a $100 \mathrm{~nm}$ ).

35 CASTELL, P., "Nanotubos de carbono", en A. CORREIA (coord.), Nanociencia y Nanotecnología en España. Un análisis de la situación presente y de las perspectivas de futuro, Phantoms foundation, España, 2011, p. 139: «Los nanotubos de carbono (NTG) son objetos nanométricos fundamentales descubiertos en 1991 por Sumio Ijima. Debido a su estructura cilíndrica singular (diámetros de unos nanómetros, longitudes de unos micrómetros y diferentes tipos de quiralidad) y a su composición (únicamente formados por átomos de carbono)». 
formática ${ }^{36}$ y nanometrología ${ }^{37}$. Esta cualidad, por supuesto, no puede entenderse como un aspecto negativo, sino todo lo contrario. Al igual que las Tecnologías de la Información y la Comunicación, la nanotecnología ha venido para cambiar nuestra sociedad en todos los ámbitos ${ }^{38}$.

Los campos más afectados por la nanotecnología son la industria, la medicina, seguridad, defensa, producción, almacenamiento de energía, medioambiente, transporte, comunicaciones, electrónica, educación y ocio ${ }^{39}$.

En lo que respecta exclusivamente a la nanomedicina, podemos concentrar su extensión en tres grandes áreas atendiendo a sus posibilidades y aplicación: sistemas de diagnóstico; liberación de fármacos; y medicina regenerativa ${ }^{40}$.

A. La nanomedicina como sistema de diagnóstico

La nanotecnología destinada al diagnóstico de enfermedades, el nanodiagnóstico, facilita la identificación de enfermedades o la predisposición a las mismas a escala celular o molecular mediante la utilización de nanodispositivos. Con anterioridad al desarrollo del nanodiagnóstico, las limitaciones de la tecnología (aún persistentes) únicamente permitían el diagnóstico cuando la enfermedad se encontraba en un estado demasiado avanzado. Por lo que la inclusión de la nanotecnología para el diagnóstico de enfermedades tiene un objetivo claro, que la identificación se pueda realizar en un desarrollo más temprano de la enfermedad ${ }^{41}$.

36 MORENO GONZÁLEZ, J. y VALENZUELA JUAN, R., "Nanotecnología aplicada a la salud", en Revista I+D, núm. 79 (2010), pp. 7-8: «La nanoinformática representa la disciplina resultante de la aplicación de la informática al campo de la nanotecnología [...]. En un futuro no muy lejano, los PCs estarán compuestos, en lugar de transistores, por otros componentes como las moléculas, neuronas, bacterias u otros métodos de transmisión de información. Entre estos proyectos se encuentra el futuro ordenador "químico", desarrollado por científicos de Hewlett-Packard y de la Universidad de California (Los Ángeles). Los circuitos de este nuevo modelo son moléculas, lo que supone transistores con un tamaño millones de veces más pequeños que los actuales».

37 PRIETO E., "Nanometrología", en A. CORREIA (coord.), Nanociencia y Nanotecnología en España. Un análisis de la situación presente y de las perspectivas de futuro, Phantoms foundation, España, 201 1, p. 113: «La nanometrología, ciencia de la medida aplicada a la nanoescala $(<100 \mathrm{~nm})$ juega pues un papel esencial en la producción de nanomateriales y dispositivos nanométricos, al permitir determinar no sólo dimeniones críticas, con incertidumbres inferiores al nanómetro, sino fuerzas, masas, propiedades ópticas, eléctricas y magnéticas, etc.».

38 ROCO, M. y BRAINBRIDGE, W. S., "Societal Implications of Nanoscience and Nanotechnology", en Kluwer Press, (2001), http://www.wtec.org/loyola/nano/NSET.Societal.Implications.

39 CORREIA, A. y SERENA, P. A. (2011: 12).

40 MUÑOZ PÉREZ, D. y BERNAL LLUCH, R. Ma ., "Análisis del impacto de la nanomedicina: consideraciones jurídicas", en Aletheia, Cuadernos Críticos del Derecho, núm. 2 (2018), pp. 7 y ss.

41 GONZÁLEZ, J. M.; LÓPEZ, M.; y RUÍZ, G., Informe de vigilancia tecnológica. Nanomedicina (vt 5), en J. DE LA SOTA RÍUS (coord.), CEIM y Dirección General de Universidades e Investigación, Madrid, 2006, p. 17. 
Las técnicas analíticas empleadas para el diagnóstico son dos: el diagnóstico in vitro y el diagnóstico in vivo. Los diagnósticos in vitro se realizan principalmente mediante nanobiosensores, que son dispositivos capaces de detectar en tiempo real, sin necesidad de marcadores fluorescentes, todo tipo de sustancias químicas y biológicas ${ }^{42}$. Además de los biosensores, se están empleando otros dispositivos de diagnóstico como los biochips o microarray de $\mathrm{ADN}^{43}$ y microarrays de proteínas ${ }^{44}$.

El nanodiagnóstico in vivo se aplica, principalmente, en el diagnóstico por imagen que permita alcanzar el nivel molecular ${ }^{45}$. Estos sistemas se basan en el uso de nanopartículas, generalmente, semiconductoras, metálicas o magnéticas, como agentes de contraste para marcaje in vivo. Permiten aumentar la sensibilidad y dan mayor contraste en las técnicas de imagen ${ }^{46}$. A este desarrollo se une el de los métodos de análisis de imágenes por ordenador, como las reconstrucciones bidimensionales y tridimensionales ${ }^{47}$.

\section{B. La nanomedicina para el suministro de fármacos}

La nanotecnología aplicada al suministro de fármacos no se centra en el medicamento en cuestión, sino en el «vehículo» o soporte que permite que dichas moléculas (las que componen el fármaco) lleguen a su destino. La idea consiste en utilizar nanoestructuras que transporten el fármaco hasta una zona concreta y, una vez reconocida la zona, lo liberen. El uso de la nanotecnología para el suministro de fármacos se denomina «nanosistemas terapéuticos»

42 Dependiendo de su sensibilidad para identificar unas u otras sustancias los biosensores se pueden clasificar, entre otros: biosensores nanofotónicos, biosensores nanoplasmónicos, o biosensores nanomecánicos. Estos nanobiosensores son tan útiles por su sensibilidad y selectividad. Además, permiten realizar análisis de sustancias en tiempo real y forma directa, a diferencia de cualquier análisis biológico o clínico que siempre requiere un marcador (ya sea fluorescente o radioactivo).

43 LÓPEZ, M., Aplicaciones de los Microrrays y Biochips en la Salud Humana, Genoma España/CIBTGUAM, p. 200: «Una serie de oligonucleótidos o fragmentos de ADN anclados a un soporte con una alta densidad espacial de fragmentos para permitir el análisis simultáneo de miles de genes. Las principales aplicaciones de los microarrays de ADN en salud humana son el seguimiento de la expresión génica, la búsqueda de compuestos activos, la medicina personalizada y la predicción de enfermedades».

44 BERTONE, P. y SNYDER, M., "Advances in functional protein microrray technology, en FEBS FOURNAL, 272, núm. 21 (2005), pp. 5300-5411. Esta tecnología tiene un gran potencial para la investigación básica en biología molecular, la búsqueda de dianas terapéuticas y la identificación de marcadores de enfermedad.

45 SAMITIER, J., "Nanomedicina: nanotecnologías aplicadas a la salud", en Dossier Científico SEBBM, núm. 168 (2011), pp. 4-5: «La imagen molecular se define como la medida, caracterización y diagnóstico de procesos biológicos celulares o moleculares in vivo, a través de imágenes generadas mediante la utilización conjunta de nuevos agentes moleculares y técnicas de imagen médica tradicionales».

46 LECHUGA, L. M. (2010: 101).

47 GONZÁLEZ, J. M.; LÓPEZ, M.; y RUÍZ, G. (2006: 20). 
A partir de la nanotecnología se han desarrollado los nuevos sistemas de liberación de fármacos (Drug Delivery Systems), que permiten que dispongamos de tratamientos más selectivos y potentes. Para la liberación de los fármacos se han propuesto una gran variedad de nanoestructuras, como nanopartículas, nanocápsulas, dendrímeros, liposomas, micelas, nanotubos, conjugados poliméricos, microgeles, etc ${ }^{48}$. Todas estas nanoestructuras son denominadas nanomateriales inteligentes ${ }^{49}$.

Las ventajas que ofrecen los nanosistemas terapéuticos son unos fármacos más selectivos y eficaces y, por lo tanto, menos tóxicos. La administración del fármaco es mucho más sencilla, puesto que pueden ser administrados por vías más cómodas para el paciente, evitando intervenciones más dolorosas o invasivas. La principal característica de los nanomedicamentos es su complejidad. En efecto, los nanomedicamentos pueden liberar moléculas terapéuticas directamente traspasando las barreras biológicas, y al mismo tiempo su coraza protectora puede incluir reactivos como anticuerpos que alcanzaran objetivos específicos ${ }^{50}$. En la actualidad y tras años de fase experimental ya se encuentran disponibles en el mercado numerosos fármacos desarrollados basados en principios de la nanotecnología ${ }^{51}$.

\section{La nanomedicina regenerativa}

La medicina regenerativa, en uso con la nanotecnología, es un área emergente muy prometedora que busca la reparación de tejidos y órganos mediante la aplicación de terapia génica, terapia celular, dosificación de sustancias biorregenerativas e ingeniería tisular ${ }^{52}$. Lo que la nanotecnología aporta es la producción de nuevos materiales y sistemas de soporte, la utilización de células madre embrionarias y adultas y la producción de moléculas bioactivas que sirvan como señales de diferenciación celular ${ }^{53}$.

En la ingeniería de tejidos, la nanotecnología puede jugar un papel predominante al facilitar nuevos materiales y técnicas que permiten una integración de los

48 LECHUGA, L. M. (2010: 108-109).

49 Para más información sobre los nanomateriales inteligentes. LOERA SERNA, S.; RUIZ ÁNGELES, J.; FLORES MORENO, J.; y SOTO PORTAS, L.; "Protegiendo fármacos con nanomateriales inteligentes", en Revista Interdisciplinaria en Nanociencia y Nanotecnología, Vol. 5, núm. 1 (2012), pp. 62-65.

50 TOMÁS QUIRINO, C. y SANCÉN CONTRERAS, F., "Ubicación de riesgos en el trabajo a nanoescala. Una visión desde la ética”, en Revista Interdisciplinar de Nanociencia y Nanotecnología, Vol. 4, núm. 1 (2011), p. 63 .

51 FERNÁNDEZ DE GATTA SÁNCHEZ, D. (dir.) y otros, Grafeno e innovación tecnológica. Aspectos jurídicos, Editorial Ratio Legis, España, 2017.

52 SAMITIER, J. (2011: 5). La ingeniería tisular combina la utilización de células vivas y biomateriales que actúan como andamiaje en la reconstrucción de tejidos realizando las funciones de la matriz extracelular.

53 SAMITIER, J. (2011: 5). 
tejidos de forma más eficiente por la posibilidad de generar microambientes más propicios para la regeneración tisular. La principal dificultad radica en encontrar materiales adecuados que permitan la fabricación de estructuras que mantengan activo el órgano afectado mientras se regenera la zona dañada. Entre los materiales que se están utilizando cabe destacar los nanotubos de carbono, las nanopartículas como hidroxiapatita o zirconia, las nanofibras de polímeros biodegradables y los nanocomposites ${ }^{54}$.

\section{GRUPO NORMATIVO REGULADOR APLICABLE A LA NANO- MEDICINA}

\section{La nanomedicina en el ordenamiento jurídico de la Unión Europea}

Ha pasado más de una década desde que la UE comenzará a adoptar medidas sobre nanociencia y nanotecnología. El 12 de mayo del 2004, la Comisión adoptó la Comunicación titulada «Hacia una estrategia europea para las nanotecnologías» ${ }^{55}$, en la que, a tenor de la imparable marcha de la nanotecnología, se proponía una estrategia que fomentase un desarrollo seguro y responsable. La UE era consciente de la importancia de esta tecnología y con esta Comunicación pretendía promover su liderazgo en la $\mathrm{I}+\mathrm{D}$ de este sector ${ }^{56}$.

La idea de focalizar esfuerzos en nanotecnología y de hacerlo, además, bajo un desarrollo responsable fue acogida favorablemente por el Consejo de Competitividad. Esto queda reflejado en las Conclusiones el 24 de septiembre de 2004, donde se insta a la Comisión a elaborar un plan de acción para la nanotecnología. El Comité Económico y Social Europeo emitió un dictamen respaldando igualmente la estrategia propuesto por la Comisión ${ }^{57}$.

54 SAMITIER, J. (2011: 5).

55 COMISIÓN DE LAS COMUNIDADES EUROPEAS, COMUNICACIÓN DE LA COMISIÓN, Hacía una estrategia europea para las nanotecnologías, Bruselas, 2004.

56 GARCÍA MARTÍNEZ, J., "Nanotecnología: un sector estratégico en innovación y creación de valor”, en Economía Exterior, núm. 44 (2008), p. 1: «En este informe se detalla que los países punteros en desarrollo nanotecnológico dedican en torno a seis euros por persona y año; la media europea se sitúa tan sólo en tres. En el vagón de cola se encuentra España con sólo cuatro céntimos de euro. Es evidente que, con estas grandes diferencias, que se muestran con crudeza en el gráfico 1, no es posible competir en condiciones favorables, a pesar de la dedicación y talento de los científicos españoles».

57 COMISIÓN EUROPEA, Comunicación de la Comisión al Consejo, al Parlamento Europeo y al Comité Económico y Social Europea. Nanociencias y nanotecnologías: Un plan de acción para Europa 2005-2009, Bruselas, 2005, p. 3 . 
El siguiente paso fue someter la propuesta de la Comisión a consulta pública el 15 de octubre de 2004, donde todas las partes interesadas fueron invitadas a presentar las observaciones pertinentes. Las conclusiones de dicha consulta han sido expuestas y descritas a través de un informe ${ }^{58}$.

Junto con estas medidas, la Comisión finaliza dando protagonismo a la seguridad y la responsabilidad del desarrollo. Invita a los inversores y profesionales a integrar una dimensión social en sus proyectos, para que los resultados puedan ser aplicables a las expectativas y preocupaciones de la población. Finalmente, cede protagonismo a la necesidad de un desarrollo que proteja la salud pública, la seguridad de los trabajadores y consumidores y el medio ambiente ${ }^{59}$.

\section{La regulación aplicable de los nanomateriales}

Primeramente, conviene destacar que no existe regulación específica sobre los nanomateriales. La UE ha optado, de momento, por ajustar y encajar este sector dentro del marco jurídico existente. Esto supone un doble reto: en primer lugar, se debe conseguir adecuar los nanoproductos en el esquema actual de regulación; en segundo lugar, se debe mantener un nivel de control elevado, lo que obliga a que aquellos que toman decisiones de control y regulación deben de tener un conocimiento técnico y científico al nivel de los que desarrollan estos productos ${ }^{60}$.

Se ha considerado que los nanomateriales se ajustan a la definición de sustancia regulada en el Reglamento (CE) n 1907/2006 del Parlamento Europeo y del Consejo de 18 de diciembre de 2006, relativo al registro, la evaluación, la autorización y la restricción de las sustancias y preparados químicos (REACH); y el Reglamento (CE)

58 Informe del Nanoforum de diciembre de 2004: http://www.nanoforum.org. En consecuencia, el 7 de junio de 2005 la Comisión publica la Comunicación «Nanociencias y nanotecnologías: Un plan de acción para Europa 2005-2009». En ella y siguiendo una línea similar a la establecida en la propuesta previa, nuevamente recomienda a los Estados miembros aumentar la inversión en I+D, así como en infraestructuras adecuadas. Prioriza la formación de todo tipo de profesionales en el sector de la nanociencia, sobre todo a tenor de su carácter interdisciplinar. E invita a los Estados miembros a fomentar esta tecnología en el sector industrial por sus favorables perspectivas en el mercado.

59 COMISIÓN DE LAS COMUNIDADES EUROPEAS, COMUNICACIÓN DE LA COMISIÓN AL PARLAMENTO EUROPEO, AL CONSEJO Y AL COMITÉ ECONÓMICO Y SOCIAL EUROPEO, Aspectos reglamentarios de los nanomateriales, Bruselas, 2008. Sobre las cuestiones jurídicas que planteaba el Plan de Acción, la Comisión tras una revisión cuidadosa de la legislación pertinente de la UE con el fin de determinar la aplicabilidad de las regulaciones existentes a los riesgos potenciales de los nanomateriales, acordó y expuso en la Comunicación de la Comisión de 17 de junio de 2008 «Aspectos reglamentarios de los nanomateriales» que, si bien el término nanomaterial no se expresa específicamente en la legislación de la UE, la legislación vigente es suficiente para proteger de los posibles riesgos sobre salud, seguridad y medio ambiente asociados a los nanomateriales.

60 HARRIS, S., "The regulation of nanomedicine: Will the existing regulatory scheme of the FDA suffice?", en Richmond Journal of Law \& Technology, Vol. 16, núm. 2 (2009), p. 19. 
$n^{\circ}$ 1272/2008 del Parlamento Europeo y del Consejo de 16 de diciembre de 2008, sobre clasificación, etiquetado y envasado de sustancias y mezclas (CLP ${ }^{61}$.

El peligro de una sustancia o mezcla es el potencial que tiene dicha sustancia o mezcla de causar daño. Depende de las propiedades intrínsecas de la misma. En este contexto, la evaluación de riesgos constituye el proceso mediante el cual se evalúa la información sobre las propiedades intrínsecas de una sustancia o mezcla con el fin de determinar su potencial de causar daño. Uno de los principales objetivos del CLP es determinar si una sustancia o mezcla presenta propiedades que lleven a clasificarla como peligrosa $^{62}$.

Los nanomateriales se encuentran en el ámbito del Reglamento REACH y debe proporcionarse información sobre sus efectos en la salud humana y en el medio ambiente. Al igual que cualquier sustancia química, algunos pueden ser tóxicos. $\mathrm{Su}$ toxicidad depende de las diversas propiedades del nanomaterial. Determinada legislación específica, como la relativa a los productos biocidas o sobre cosméticos, también contempla la necesidad de información sobre los nanomateriales ${ }^{63}$.

La Agencia Europea de Sustancias y Mezclas Químicas (ECHA) colabora con las autoridades competentes de los Estados miembros, la Comisión Europea, organizaciones internacionales, la industria y otras partes interesadas ${ }^{64}$. En sus funciones,

61 CLP es la abreviatura de la expresión inglesa "Classification, Labelling and Packaging» o «clasificación, etiquetado y envasado». El Reglamento CLP entró en vigor en enero de 2009, y el método de clasificación y etiquetado de sustancias químicas que introdujo se basa en el Sistema Globalmente Armonizado (SGA) de las Naciones Unidas. El Reglamento sustituye progresivamente a dos actos legislativos anteriores, a saber, la Directiva «Sustancias peligrosas» (DSD) y la Directiva «Preparados peligrosos» (DPD). Se estableció un período transitorio hasta 2015. El Reglamento CLP se atiene a diversas formulaciones en virtud de las cuales la Unión confirma su intención de contribuir a la armonización global de los criterios de clasificación y etiquetado mediante la incorporación a la legislación de la Unión de los criterios del Sistema Globalmente Armonizado de Clasificación y Etiquetado de productos químicos (SGA) internacionalmente acordados.

62 Recomendación 2011/696/UE de 18 de octubre, relativa a la definición de nanomaterial. Téngase en cuenta que, siempre que en este documento de orientación aparezca una discusión sobre «sustancias y mezclas», también abarca aquellos «artículos específicos determinados» sujetos a clasificación de acuerdo con la parte 2 del anexo I del CLP. Esto ha sido complementado en 201 1, mediante una recomendación de la Comisión Europea sobre la definición de nanomaterial que ha quedado incluida en el REACH y el CLP.

63 A esta conclusión llega la Agencia Europea de Sustancias y Mezclas Químicas, como recoge en su web oficial: http://www.echa.europa.eu/chemicals-in-our-life/hot-scientific-topics/nanotechnology.

64 EUROPEAN CHEMICALS AGENCY, ECHA Services at a glance, 2014: «La Agencia Europea de Sustancias y Mezclas Químicas (ECHA) será el timón de las autoridades reguladoras a la hora de aplicar la pionera legislación de la UE sobre productos químicos en pro de la salud humana y el medio ambiente, así como de la innovación y la competitividad. La ECHA asesora a las empresas en el cumplimiento de la legislación, aboga por el uso seguro de los productos químicos, brinda información sobre los productos químicos y se ocupa de los productos químicos que suscitan inquietud». 
la ECHA es la competente para registrar, evaluar, autorizar o restringir los nanomateriales (de acuerdo la REACH), y, por otro lado, clasificar y etiquetarlos (de acuerdo con la CLP), como lo haría con cualquier otra sustancia química.

Finalmente, indicar que la ECHA planea sus actividades por medio de programas de trabajo plurianuales (PTPAs) y de programas de trabajo anuales (PTs). Los informes generales dan cuenta de las actividades de la agencia durante un año determinado ${ }^{65}$.

\section{La regulación aplicable a la nanomedicina}

En lo que respecta al marco jurídico, la nanomedicina presenta una enorme complejidad. Hasta el momento no existe una regulación específica en materia de nanomedicina en la Unión Europea (ni siquiera existe una regulación más genérica que abarque todo el sector de la nanotecnología). Asimismo, como aspecto más destacable, el actual ordenamiento jurídico de la UE carece de suficientes mecanismos que le permitan adecuarse a la regulación de este sector.

La falta de un marco jurídico elaborado específicamente para regular la nanomedicina, y la amplia diversidad de este sector provocan que el número de normas aplicables sea muy amplio y heterogéneo. Caben destacar las siguientes:

- Productos médicos. Un elenco normativo que, en mayor o menor medida, afecta a la nanomedicina son aquellas normas (Directivas en su mayoría) desarrolladas para regular los productos médicos. De este modo, la nanomedicina queda jurídicamente vinculada a la Reglamento (CE) n 726/2004 del Parlamento Europeo y del Consejo de 31 de marzo de 2004 por el que se establecen procedimientos comunitarios para la autorización y el control de los medicamentos de uso humano y veterinario y por el que se crea la Agencia Europea de Medicamentos ${ }^{66}$; la Directiva 2003/63/CE, por la que se establece un código comunitario sobre medicamentos para uso humano; la Directiva 2003/94/UE, por la que se establecen los principios y directrices de las prácticas correctas de fabricación de los medicamentos de uso humano y de los medicamentos en investigación de uso humano; y, con carácter especial, la Directiva

65 Al principio la ECHA tenía un PTPA trienal revisado anualmente, con períodos de planificación consecutivos que cubrían el año en curso, el siguiente y el anterior. A partir de 2014 la ECHA tiene una estrategia a medio plazo de cinco años descrita en el PTPA 2014-2018. El nuevo proyecto de PTPA 2014-2018 se presentó a consulta pública en 2013. Los PT anuales recogen los objetivos de la ECHA para el año en cuestión, cuantificados en función de indicadores específicos, así como las metas establecidas con carácter anual. A finales de octubre de cada año, el Consejo de Administración de la ECHA aprueba un PT anual de la Agencia para el año posterior. Vide EUROPEAN CHEMICALS AGENCY, Programa de trabajo 2019. Aspectos destacados, 2019, pp. 1-2.

66 Disponible en el siguiente enlace: Vide http://ec.europa.eu/health//sites/health/files/files/ eudralex/vol-1/reg_2004_726/reg_2004_726_en.pdf. 
2001/83/UE, por la que se establece un código comunitario sobre medicamentos para uso humano.

- Dispositivos médicos ${ }^{67}$. La heterogeneidad de los productos en el área de la nanomedicina conlleva que algunos de estos puedan ser enmarcados dentro del concepto «dispositivo médico». Es la Directiva 93/42/CEE, relativa a los productos sanitarios, la que establece el concepto de dispositivos médicos y regula dicho sector. Junto a esta norma hay que destacar la Directiva 90/385/CEE, relativa a la aproximación de las legislaciones de los Estados miembros sobre los productos sanitarios implantables activos; y la Directiva 98/79/CE sobre productos sanitarios para diagnóstico in vitro.

- Productos $\operatorname{cosméticos}^{68}$. Otro conjunto de normas son las diseñadas para regular los productos cosméticos. Para ello, en primer lugar, se aprobó la Directiva 76/768/ CEE, que define «producto cosmético» como «toda sustancia preparada destinada a ser puesta en contacto con las diversas partes superficiales del cuerpo humano (epidermis, sistemas piloso y capilar, uñas, labios y órganos genitales externos) o con los dientes y o las mucosas bucales, con el fin exclusivo o principal de limpiarlos y protegerlos para mantenerlos en buen estado, modificar su aspecto o corregir los olores corporales» (art. 1) ${ }^{69}$.

El Reglamento (CE) n 1223/2009 sobre los productos cosméticos que entró en vigor el 11 de julio de 2013 (deroga la Directiva 76/768/CE), surge con el fin de velar por un alto nivel de protección de los consumidores, la libre circulación de mercancías y la seguridad jurídica de los fabricantes, así como alcanzar un alto nivel de protección de la salud humana con respecto a todo producto cosmético que contenga nanomateriales (art. 16.1). Para ello establece medidas como la obligatoriedad del productor del cosmético de presentar información previa a la Comisión en el caso de que el producto presente sustancias en forma de nanomateriales [art. 13.1.f)]..$^{70}$

El Reglamento sobre productos cosméticos ha sido desarrollado posteriormente mediante el Reglamento UE n ${ }^{\circ}$ 655/2013, por el que se establecen los criterios

67 TOMA-BIANOV, A., "European Union Regulations on nanomedicine", en Bulletin of the Transilvania University of Brasov, Vol. 5 (54), núm. 2 (2012), p. 106.

68 TOMA-BIANOV, A. (2012: 106-107).

69 En la actualidad, no existe ninguna regulación específica sobre la nanotoxicidad en ningún país del mundo. El problema se incrementa cuando tras años de investigación comienzan a salir al mercado numerosos productos compuestos por nanomateriales. Según Woodrow Center for Scholars, ya existen más de 1.800 líneas de productos de nanotecnología en el mercado generando miles de millones de euros. Hasta ahora el procedimiento empleado por la Unión Europea se basa en exigir el etiquetado de los alimentos, cosméticos y biocidas que contengan nanopartículas, lo que significa que si un país decide exportar estos productos al territorio europeo deben etiquetarlos adecuadamente. Algunos países en concreto (Francia, Bélgica, Dinamarca, Noruega, Suecia, Canadá, China) exigen un registro de estos productos donde se declara las características y las propiedades.

70 JIMÉNEZ SIMÓN, N., "Productos cosméticos: marco normativo y prevención de riesgos laborales", en Notas Técnicas de Prevención (NTP), 1074 (2016), p. 1. 
comunes a los que deben responder las reivindicaciones relativas a los productos cosméticos; y la Decisión de Ejecución de la Comisión de 25 de noviembre de 2013 sobre las directrices relativas al anexo I del Reglamento (CE) no 1223/200971.

- Productos químicos ${ }^{72}$. Este tipo de productos están sujetos a una serie de normas concernientes al Registro, Evaluación, Autorización y Restricción de los Productos Químicos (REACH son las siglas en inglés), las cuales han presentado un cambio en el actual sistema regulatorio, asignando la carga del riesgo al fabricante en vez de a las autoridades, ampliando el alcance de los registros de los productos químicos, reemplazando los sistemas de registro descentralizados por un sistema central europeo, y sustituyendo todo el conjunto de normas por un sistema de regulación único.

- Directrices y buenas prácticas en la fabricación de medicamentos. Este grupo normativo está formado por dos Directivas: la Directiva 2001/20/CE, relativa a la aproximación de las disposiciones legales, reglamentarias y administrativas de los Estados miembros sobre la aplicación de buenas prácticas clínicas en la realización de ensayos clínicos de medicamentos de uso humano; y la Directiva 2003/94/CE, por la que se establecen los principios y directrices de las prácticas correctas de fabricación de los medicamentos de uso humano y de los medicamentos en investigación de uso humano.

Con la aprobación del Reglamento (UE) n ${ }^{\circ}$ 536/2014 del Parlamento Europeo y del Consejo de 16 de abril de 2014, sobre los ensayos clínicos de medicamentos de uso humano ${ }^{73}$, se deroga la Directiva 2001/20/CE. Esta norma se aplica a todos los ensayos clínicos realizados en la UE (art. 2) y aporta una nueva definición de «ensayo clínico» introduciendo un concepto más amplio de «estudio clínico» ${ }^{74}$. Si bien no hace alusión a ningún término «nano», es evidente su vinculación con este ámbito.

71 JIMÉNEZ SIMÓN, N. (2016: 1).

72 TOMA-BIANOV, A. (2012: 107).

73 SANZ CEBALLOS, L.; FERRIT MARTÍN, M.; y otros, "Novedades introducidas por el nuevo Real Decreto legislativo 1090/2015 de ensayos clínicos con medicamentos en España”, en Ars Pharm., 57, núm. 4 (2016), p. 148. En respuesta al Reglamento 536/2014, se aprobó en España el Real Decreto 1090/2015, de 4 de diciembre, por el que se regulan los ensayos clínicos con medicamentos, los Comités de Ética de la Investigación con medicamentos y el Registro Español de Estudios Clínicos. El nuevo RD 1090/2015, «introduce modificaciones sustanciales en la legislación nacional con el objetivo de hacer posible la aplicación del nuevo Reglamento 536/2014 del Parlamento Europeo y del Consejo sobre Ensayos clínicos de medicamentos de uso humano. Sus pilares serán la simplificación de procedimientos, el acortamiento de plazos, la exigencia de transparencia de los resultados, el establecimiento de un marco armonizado de autorización con un sistema único de evaluación y el establecimiento de mecanismos de cooperación transfronteriza permitiendo la participación de pacientes en EC de otros Estados, especialmente interesante en la investigación clínica de medicamentos huérfanos para el tratamiento de patologías poco frecuentes».

74 GARCÍA VIDAL, A., "Nuevo reglamento de la Unión Europea sobre los ensayos clínicos de medicamentos de uso humano", en Análisis Farmacéutico GA \&P, (2014), p. 1. 
En definitiva, delimitar el grupo normativo regulador aplicable a la nanomedicina supone una labor compleja como consecuencia de dos factores principalmente: en primer lugar, la falta de una norma reguladora específica; y, en segundo lugar, el amplio campo de actuación y aplicación de la nanomedicina le hacen abarcar múltiples áreas de desarrollo ${ }^{75}$.

\section{G. European Technology Platform Nanomedicine}

La ETP nanomedicine es una iniciativa liderada por el sector industrial para establecer, junto con la Comisión Europea, la aplicación de la nanotecnología con el fin de lograr avances en la asistencia sanitaria. Ha sido desarrollada para apoyar a sus miembros, mejorar la comunicación entre ellos y responsabilizarse de la coordinación de sus investigaciones ${ }^{76}$. El objetivo es trabajar en conjunto con la Comisión Europea y los Estados miembros, para ello colabora con determinadas plataformas nacionales ${ }^{77}$.

La ETP fue originada por un grupo de 53 actores europeos, integrado por expertos industriales y académicos, estableció en 2005 una Plataforma Tecnológica

75 Junto con todos estos grupos normativos, podemos destacar otra serie de normas que, con una menor influencia, pueden ser vinculadas con la nanomedicina: Regulación de productos específicos: Reglamento (CE) no 1394/2007 sobre medicamentos de terapia avanzada; Reglamento (CE) $\mathrm{n}^{\circ}$ 1901/2006 sobre medicamentos para uso pediátrico; y Reglamento (CE) n ${ }^{\circ}$ 141/2000 sobre medicamentos huérfanos. Directiva 98/44/CE relativa a la protección jurídica de las invenciones biotecnológicas. Organismos modificados genéticamente: Directiva 2001/18/CE sobre la liberación intencional en el medio ambiente de organismos modificados genéticamente; Reglamento (CE) n 1946/2003 relativo al movimiento transfronterizo de organismos modificados genéticamente. Patentes: Directiva 98/44/CE, del Parlamento Europeo y del Consejo, de 6 de julio, relativa a la protección jurídica de las invenciones biotecnológicas; Reglamento (CEE) n $n^{\circ}$ 1768/92 del Consejo, relativo al certificado complementario de protección para los medicamentos (versión codificada aprobada por Reglamento(CE) n 469/2009 del Parlamento Europeo y del Consejo, de 6 de mayo de 2009); Reglamento (CE) n 1610/96 del Parlamento Europeo y del Consejo, de 23 de julio de 1996, por el que se crea un certificado complementario de protección para los productos fitosanitarios; Reglamento (CE) no 816/2006 del Parlamento Europeo y del Consejo, de 17 de mayo de 2006, sobre la concesión de licencias obligatorias sobre patentes relativas a la fabricación de productos farmacéuticos destinados a la exportación a países con problemas de salud pública.

76 Las instituciones que componen la ETP nanomedicine son numerosas y abarcan diferentes áreas de investigación: industrial (empresas como NANOBIOTIX o ONXEO S.A.); académicas (como INEB, Instituto de Ingeniería Biomedica o IMDEA Nanociencia); o dedicadas a la salud (como University Medical Center Utrecht). Un total de casi 150 instituciones participan en este proyecto, entre los que se encuentra la Comisión Europea. En España, por ejemplo, participa el Instituto de Salud Carlos III. Vide http://www.etp-nanomedicine.eu/public/about/members/list_etp?type=All.

77 La ETP Nanomedicine está trabajando en estrecha colaboración con una serie de instituciones nacionales: British Society for Nanomedicine, Nano-Med Czech Republic, Deutsche Plattform NanoMedizine, French Society for Nanomedicine, Greek Nanomedicine Platform, Nanomedicine theme within NanoNextNL (Países Bajos), Norwegian NanoMedicine Network, Romanian Nanomedicine Network (RO-NANOMED), NanoMed Northe, Spanish Nanomedicine Plartform (como representante de España). 
Europea de Nanomedicina ${ }^{78}$. La primera tarea de este grupo de alto nivel era escribir un documento de visión para esta área altamente orientada hacia el futuro de la asistencia sanitaria basada en la nanotecnología en el que los expertos describen una extrapolación de las necesidades y posibilidades hasta el año 2020. A partir de 2006 esta Plataforma se ha abierto a una amplia participación y ha emitido una serie de documentos estratégicos que muestran una bien elaborada manera europea común de trabajar juntos para el cuidado de la salud del futuro tratando de coincidir con las altas expectativas de que la nanomedicina se ha planteado hasta ahora.

\section{La nanomedicina en el ordenamiento jurídico español}

España destaca por ser referente en materia de nanotecnología. En 2011 se situaba como séptimo país del mundo en términos de producción científica en este ámbito y el más productivo en el contexto iberoamericano ${ }^{79}$.

Como veremos a continuación, la inversión y participación del sector público ha tenido una importante influencia, pero probablemente el factor clave que ha determinado el desarrollo de la nanotecnología en España ha sido una rápida autoorganización de los científicos en redes como la Red Nanociencia (1999-2004) y la Red NanoSpain ${ }^{80}$.

La Red NanoSpain persigue como objetivo más ambicioso el fortalecimiento y la divulgación de la Nanociencia y la Nanotecnología española en el Espacio Europeo de investigación e innovación. Así, la Red NanoSpain fomenta la transferencia de conocimientos entre los grupos que la conforman y facilita el intercambio, lo que se traduce en una mejora en la cooperación entre los agentes implicados en el sector de la nanotecnología ${ }^{81}$.

78 En Estados Unidos, principal potencia mundial en el ámbito de la nanotecnología, la Oficina Nacional de Coordinación de Nanotecnología (NNCO, siglas en inglés) es la responsable de proporcionar apoyo técnico y administrativo en materia de nanociencia. El subcomité de Ingeniería y Tecnología (NSET) sirve como punto central de contacto para las actividades federales de nanotecnología, y proporciona alcance público en nombre de la Iniciativa Nacional de Nanotecnología. El NNCO coordina la preparación y publicación de la Iniciativa Nacional de Nanotecnología (NNI, siglas en inglés) planificación interinstitucional, el presupuesto y los documentos de evaluación, como el suplemento anual NNI para el presupuesto del presidente. La oficina organiza sesiones de la Subcomisión NSET y sus grupos de trabajo.

79 ORGANIZACIÓN DE ESTADOS IBEROAMERICANOS, La nanotecnología en Iberoamérica. Situación actual y tendencias, 2009, http://www.oei.es/salactsi/nano.pdf.

80 SERENA, P. A., y TUTOR, J. D., "La divulgación y la formación de la nanociencia y la nanotecnología en España: un largo camino por delante", en Revista Interdisciplinar de Nanociencia y Nanotecnología, Vol. 4, núm. 2 (2011), p. 49.

81 MORENO MOLINA, A. M., "Productos químicos. Biotecnología. Acuerdos Voluntarios", en J. PICÓN RISQUEZ (coord.), Derecho medioambiental de la Unión Europea, McGraw-Hill Interamericana de España, España, 1996, pp. 273-296. El apoyo del sector público también ha sido determinante para el desarrollo de la nanotecnología. La presión ejercida por la Red NanoSpain se trasladó hasta el Plan Nacional 
Desde el Plan Nacional de I-D-i (PNIDI) 2004-2007, la AENN ha estado presente en los Planes de Trabajo de las convocatorias 2004 a 2007. Además, ha tenido un papel protagonista en el PNIDI 2008-201182 y el PNIDI 2013-2016. Con independencia de la PNIDI, cabe destacar el Plan Ingenio 2010, que incluye los programas Consolider, CIBER y CENIT. Junto a estos programas se han financiado grandes infraestructuras como el sincrotrón $\mathrm{ALBA}^{83}$.

No obstante, la Administración Central no ha sido la única que ha hecho un esfuerzo económico para el desarrollo de la nanociencia y la nanotecnología, debe ser destacado igualmente el esfuerzo realizado por las CGAA. La gran mayoría de las empresas, fundaciones, centros tecnológicos y de investigación se encuentran en la Comunidad Autónoma de Madrid, Cataluña y País Vasco ${ }^{84}$. En menor medida cabe destacar las regiones de Andalucía y Comunidad Valenciana.

de I-D-i (PNIDI) 2004-2007 donde por primera vez se incluyó la Acción Estratégica de Nanociencia y Nanotecnología (AENN). Las líneas de actuación de la AENN han sido muy amplias, acorde con el carácter multidisciplinar de la nanotecnología: biotecnología, biomedicina, agroalimentación, energía, medioambiente, almacenamiento magnético de información, magnetoeletrónica, nanoelectrónica, optoelectrónica, nanoestructuras semiconductoras, dispositivos y máquinas nanométricas, nanomanipulación, nanocaracterización y materiales nanocompuestos.

82 VIDAL CORREA, L., y ZAYAGO LAU, E., "Nanomedicina: expectativas y regulación para el desarrollo", en Observatorio del desarrollo, Vol. 2, núm. 8 (2014), p. 19: «El nuevo PNIDI se plantea como una oportunidad para cambiar de un enfoque temático a un enfoque instrumental. Los objetivos generales del PNIDI 2008-2011 son: (i) situar a España en la vanguardia del conocimiento; (ii) promover un tejido empresarial altamente competitivo; (iii) desarrollar una política integral de ciencia, tecnología e innovación, imbricando a los ámbitos regionales en el sistema de ciencia y tecnología; (iv) avanzar en la dimensión internacional como base para el salto cualitativo del sistema; (v) conseguir un entorno favorable a la inversión en $\mathrm{I}+\mathrm{D}+\mathrm{i}$; y (vi) fomentar la cultura científica y tecnológica de la sociedad».

83 Vide https://www.cells.es/es/que-es-alba/bienvenida (web oficial): «El Sincrotrón ALBA es una infraestructura científica de tercera generación situada en Cerdayola del Vallés (Barcelona) y es la más importante de la zona del Mediterráneo. Se trata de un complejo de aceleradores de electrones para producir luz de sincrotrón, que permite visualizar la estructura atómica y molecular de los materiales y estudiar sus propiedades».

84 VIDAL CORREA, L., y ZAYAGO LAU, E. (2014: 20-21): «El País Vasco lleva varios años apostando por la investigación en nanociencia y nanotecnología, que ya aparecían como áreas prioritarias del Plan de Ciencia, Tecnología e Innovación 2001-2004 del Gobierno Vasco. El principal instrumento desde el que se ha planteado la investigación en nanociencia y nanotecnología, ha sido el Programa de Investigación Estratégica ETORTEK, en cuyo marco se han ejecutado en el periodo 2005-2007 dos grandes proyectos en los últimos 5 años: NANOMATERIALES, centrado en la generación de conocimiento en el campo de los nanomateriales desde una perspectiva de la ciencia e ingeniería de materiales, y NANOTRON, centrado en la generación de conocimiento en nanosistemas y nanoelectrónica. La culminación de las iniciativas de investigación en nanociencia y nanotecnología en el País Vasco ha sido la concesión, en el marco del Programa Consolider-Ingenio 2010, del Proyecto "CIC nanoGUNE Consolider" orientado hacia la puesta en marcha del CIC nanoGUNE como nuevo centro de I+D en nanociencia y nanotecnología [37]. Sin embargo, no todo termina aquí, y el Gobierno Vasco, ha diseñado la Estrategia nanoBASQUE 2015, orientada a promocionar la nanotecnología hacia el ámbito empresarial y así incrementar la compe- 
En Cataluña se ha llevado a cabo una importante iniciativa mediante la Acción Especial de desarrollo de la Nanociencia y Nanotecnología, financiando estancias posdoctorales en el Laboratorio de Investigación en Nanobioingeniería (CREBEC) perteneciente al Instituto Catalán de Bioingeniería (IBEC) y el Instituto Catalán de Nanotecnología.

Cabe destacar el centro IMDEA Nanociencia, en Madrid, apoyado por el gobierno de la Comunidad Autónoma, el centro CIC-nanoGUNE en el País Vasco, el Instituto de Nanociencia de Aragón o la Unidad de Nanotecnología de la Universidad de Oviedo.

Por otro lado, a partir del denominado Eje-Nano dentro del Plan Estratégico, se ha fomentado el desarrollo de nuevos centros como el Instituto de Nanotecnología de Asturias y el Centro de Investigación en Nanociencia y Nanotecnología ubicado en Barcelona en colaboración con el Gobierno de Cataluña. En Andalucía se han creado el Centro Andaluz NIBC y el Centro Andaluz de Biotecnología y Nanomedicina $(\mathrm{BIONAND})^{85}$.

\section{LOS RETOS JURÍDICOS QUE PLANTEA LA NANOMEDICINA}

La tecnología ha proporcionado enormes avances y ventajas en todos los ámbitos y a diferentes escalas, transformando nuestra sociedad. No obstante, el avance tecnológico frenético del que somos testigos es una evolución de luces y sombras.

En la actualidad, y desde hace bastantes años, nuestra sociedad ha pasado de estar sometida a los daños generados por la naturaleza, a verse afectada principalmente por daños generados por la propia tecnología que desarrollamos. Esta afirma-

titividad industrial del País Vasco. El resultado global que se espera obtener para el año 2015 es el de contar con una comunidad de conocimiento con una masa crítica investigadora consolidada y un nuevo sector económico competitivo posibilitado por la nanotecnología. Uno de los pilares fundamentales sobre los que se asienta la Estrategia nanoBASQUE 2015 es, el ya mencionado, Centro de Investigación Cooperativa en Nanociencias - CIC nanoGUNE, que desarrolla el concepto de investigación cooperativa, ya puesto en práctica con éxito en varios casos en la Comunidad Autónoma del País Vasco».

85 El nuevo Centro Andaluz de Nanomedicina y Biotecnología (BIONAND) es un centro de carácter mixto participado por la Consejería de Salud y Bienestar Social, la Consejería de Economía, Innovación, Ciencia y Empleo, y la Universidad de Málaga, concebido como espacio para la investigación de excelencia en nanomedicina. BIONAND ha sido diseñado, como un centro de carácter multidisciplinar, en el que la interacción de diferentes profesionales procedentes del ámbito sanitario, universitario y empresarial permitirá generar nuevos sistemas de diagnóstico, prevención y tratamiento de enfermedades a partir de la creación y desarrollo de dispositivos, materiales y abordajes a escala nanométrica. Este centro se enmarca en el Programa Andaluz de Investigación en Nanomedicina, uno de los tres programas de investigación en Terapias Avanzadas de la Comunidad Autónoma de Andalucía, junto con el Programa de Investigación en Terapia Celular y Medicina Regenerativa, y el de Genética Clínica y Medicina Genómica. 
ción es la que ocupa una parte muy importante en la investigación científica, valorar los beneficios que nos aporta una tecnología frente a los riesgos a los que nos expone. Si bien, en la gran mayoría de los casos los enormes éxitos que genera el desarrollo tecnológico supera los riesgos que provoca.

De este modo, el desarrollo tecnológico se estima como el sustento básico de la evaluación social, con todas las ventajas que supone, pero también con todos los peligros que engloba. Lo que en la doctrina se conoce como la sociedad del riesgo ${ }^{86}$.

Ahora bien, los daños ocasionados por los desastres naturales se generan a partir de fenómenos incontrolables y en ocasiones impredecibles, de los que no se desprende responsabilidad alguna. Por el contrario, detrás de cualquier tecnología se encuentran decisiones humanas y, por lo tanto, cualquier daño causado queda bajo responsabilidad ${ }^{87}$. En este tipo de daños generados por la tecnología el Derecho adquiere un enorme protagonismo. Como indica Esteve Pardo, cualquier riesgo que emane de la tecnología debe quedar bajo el control del Derecho ${ }^{88}$.

El impresionante despliegue de la ciencia y la tecnología que caracteriza nuestros días obliga al Derecho a desarrollarse para permitirle abarcar situaciones cada vez más numerosas y complejas ${ }^{89}$.

El Derecho debe establecer quién adopta las decisiones y bajo qué responsabilidad. Además, debe pretender siempre un equilibrio entre el riesgo para la salud o para el sector industrial que diseña la tecnología. Este control sin duda puede suponer una ralentización en el desarrollo tecnológico, pero no presume que todo el proceso deba ser puesto en duda o que se criminalice cualquier avance ${ }^{90}$.

La regulación plena de la sociedad tecnológica actual es inalcanzable, ya que existe siempre un margen de incertidumbre asociado a las nuevas tecnologías. Es más, nos encontramos ante un desarrollo tecnológico cada vez más rápido y por lo tanto ante un riesgo en constante aumento. Por ello, la ciencia tiene la función de dar

86 BECK, U., Risikogesellschaft. Auf dem Weg in eine andere Moderne, Frankfurt am Main, 1986.

87 ESTEVE PARDO, J., "Ciencia y Derecho ante los riesgos para la salud. Evaluación, decisión y gestión”, en Documentación Administrativa, núm. 265-266 (2003), p. 138. ESTEVE PARDO, J., "Convivir con el riesgo. La determinación del riesgo permitido", en E. J. PÉREZ ALONSO, E. ARANA GARCÍA, J. L. SERRANO MORENO y P. MERCADO PACHECO, Derecho, globalización, riesgo y medio ambiente, Tirant lo Blanch, España, 2012, pp. 275 y ss. ESTEVE PARDO, J., "Responsabilidad de la Administración y riesgos del desarrollo", en A. B. CASARES MARCOS y T. QUINTANA LÓPEZ (dirs.), La responsabilidad patrimonial de la Administración Pública: estudio general y ámbitos sectoriales, Vol. 2, Tirant lo Blanch, España, 2013, pp. 1549 y ss.

88 ESTEVE PARDO, J. (2003: 138).

89 MONTANO, P. J., "La responsabilidad de médicos y científicos ante las nuevas tecnologías biogenéticas", en Cuadernos de Bioética, núm. 1 (1995), p. 81.

90 ESTEVE PARDO, J. (2003: 138). 
a conocer y valorar los riesgos. No obstante, es el Derecho el que debe decidir sobre la incertidumbre científica. En definitiva, es la ciencia la que informa sobre los posibles riesgos y el Derecho el que debe adoptar decisiones.

\section{EL DESARROLLO DE UN GRUPO NORMATIVO REGULADOR APLICABLE A LA NANOMEDIGINA}

La necesidad de un marco jurídico regulador de la nanomedicina y la nanociencia se justifica en numerosos estudios científicos que alertan de los posibles riesgos derivados de los nanomateriales. Esto supone el desarrollo de normas que controlen los ensayos clínicos con nanomateriales, y otras que controlen la comercialización de productos compuestos por los mismos.

Hasta ahora el marco jurídico regulador de la nanomedicina en las dos grandes potencias en este sector, EE.UU. ${ }^{91}$ y UE, se ha basado en la adaptación del marco normativo existente. Se ha considerado que los cuerpos legales son apropiados para tratar esta tecnología y por lo tanto suficientes. Al respecto, con carácter general, se está empleando el marco legal aplicable a los químicos para tratar los nanomateria$\operatorname{les}^{92}$.

En la situación actual se han dado cuatro factores que invitan a plantear la posibilidad de desarrollar una apropiada regulación que abarque de forma genérica el sector o, por lo menos, determine aspectos clave, como son los productos compuestos por nanomateriales (en los que se integrarían los nanomedicamentos) ${ }^{93}$.

En primer lugar, aunque nos encontramos ante un sector complejo e interdisciplinar, se le ha dedicado una amplia cantidad de tiempo y presupuesto, y la información que tenemos sobre el mundo «nano» está en constante crecimiento. Puede que el conocimiento científico actual sea suficiente para iniciar el proceso regulador.

91 BAWA, R., "FDA and Nanotech: Baby steps Lead to Regulatory Uncertainty, en H. MORIYAMA y F. SHAHIDI, Bio-Nanotechnology: A revolution in Food, Biomedical and Health Sciences, Edita Debasis John Wiley \& Sons, Nueva York, 2013, p. 723. La FDA en 2007 creó un grupo de trabajo diseñado para elaborar un informe explicativo de la nanotecnología, es este informe se concluyó que el marco jurídico de los EE.UU. era suficiente para garantizar la seguridad adecuada.

92 FOLADORI, G., y INVERNIZZI, N., "La regulación de las nanotecnologías: una mirada desde las diferentes EUA-UE", en Vigil. Sanit. Debate, 4, núm. 2 (2016), p. 10.

93 COMISIÓN EUROPEA, Communication from the Commission to the European Parliament, the Council and the European Economic and Social Committee. Regulatory aspects of nanomaterials, Bruselas, 2008, pp. 4-8. En esta Recomendación la Comisión Europea destaca 4 factores clave para la regulación de la nanotecnología: los químicos, la protección de los trabajadores, los productos compuestos de nanomateriales y la protección medioambiental. 
En segundo lugar, el desarrollo de esta tecnología se sitúa en una fase en la que tras años de investigación comienzan a comercializarse múltiples productos. La ausencia de una regulación específica genera inseguridad al consumidor y problemas conceptuales muy básicos. En casos concretos se desconoce que normativa aplicar tanto para su producción como para su comercialización. Esto además está generando conflictos sobre patentes ${ }^{94}$.

En tercer lugar, son evidentes e innegables los beneficios y los innumerables usos que puede aportar la nanomedicina a la sociedad, sin embargo, son numerosos los estudios que alertan sobre los posibles riesgos que genera su uso, tanto para la salud pública como para el medioambiente ${ }^{95}$.

Finalmente, la importancia y los enormes beneficios que puede aportar la nanomedicina requiere de un marco legal que permite redistribuir la igualdad en el acceso a esta tecnología. Se están desarrollando estudios sobre la eficacia de este sector en enfermedades tan graves como el cáncer y los resultados están siendo muy satisfactorios. Por lo expuesto, debe ser prioritario que estos tratamientos se apliquen de un modo igualitario y no discriminatorio ${ }^{96}$.

Estas cuestiones están despertando cierta inquietud y pueden determinar un arduo y lento, pero necesario, proceso de regulación. Hasta ahora el marco legal sobre el que se está sustentando se ha visto claramente rebasado por los desarrollos nanotecnológicos y su comercialización ${ }^{97}$. Tanto las instituciones como las normas han demostrado ser endebles e inadecuadas: las instituciones en muchos casos por carecer de recursos suficientes; y las leyes por reflejar importantes lagunas. La conclusión ha quedado más que evidente, la situación actual requiere de una amplia regulación del sector de la nanociencia y la nanomedicina que abarque, cuanto menos, temas tan importantes como la comercialización de los productos como los posibles riesgos de la nanotoxicidad tanto para consumidores como para los propios investigadores.

En su espectro más amplio, la nanotecnología es una tecnología habilitadora que amplía el potencial de otras técnicas y ciencias. Actualmente, la nanotecnología tiene aplicaciones en todos los sectores productivos, aunque sobresalen el automotriz, semiconductores, construcción, químico y médico ${ }^{98}$. Esta diversidad y heterogeneidad son, sin ninguna duda, muy positivas, pero pueden generar alguna duda al legislador.

94 SANDERSON, K., "Putting nanotechnology regulation under the microscope", en The Guardian, 24 de junio de 2013.

95 SANDERSON, K. (2013).

96 VIDAL CORREA, L. y ZAYAGO LAU, E. (2014: 34).

97 VIDAL CORREA, L. y ZAYAGO LAU, E. (2014: 34).

98 VIDAL CORREA, L. y ZAYAGO LAU, E. (2014: 31). 
La primera cuestión a la que debe hacer frente el legislador es decidir qué tipo de regulación llevar a cabo, una norma que abarque a todo el sector de la nanotecnología en su conjunto, o desarrollar diferentes normas según un enfoque sector a sector. Lo ideal es diseñar un conjunto de normas especializadas en cada sector que permitan una regulación individualizada, ajustándose a las tecnologías propias de cada área que pueden ser totalmente divergentes. La política reguladora debería ser capaz de regular los retos de cada sector, que en el caso de la nanomedicina son múltiples y muy relevantes.

La segunda cuestión a tratar es la intensidad de la regulación. Se puede optar por una norma rígida que abarque todos los aspectos posibles, o, por el contrario, por una norma más flexible. Una regulación más exigente supondría un lastre para la industria y, por lo tanto, un retraso en el desarrollo de la tecnología a nanoescala. Sin embargo, una regulación más rígida sería esencial para solventar los problemas de salud pública y medioambiente ya detectados, o futuros inconvenientes. Una regulación flexible permitiría además una adaptación más sencilla de la norma a los continuos avances tecnológicos. En este sentido, debemos entender que hasta ahora el marco legal se ha visto rebasado por los desarrollos nanotecnológicos y su comercialización, es más, no podemos descartar futuros avances totalmente impredecibles a corto plazo para la ciencia, aún más para el derecho. Lo ideal sería una norma o grupo de normas que, como poco, establecieran un fuerte control sobre la toxicología de los nanomateriales, que afronte muy seriamente los efectos nocivos que estos puedan tener sobre la salud o el medio ambiente.

A. La investigación como elemento esencial para una adecuada regulación

La regulación debe estar sustentada por un amplio conocimiento científico. Las actividades de investigación están en curso bajo los programas marco de I+D y el Centro Común de Investigación.

En particular, se necesita investigación en áreas que amplíen la evaluación y la gestión de riesgos, tales como ${ }^{99}$ : los datos sobre los efectos tóxicos y ecotóxicos, así como métodos de ensayo para generar estos datos; los datos sobre los usos y exposiciones en todo el ciclo de vida de los nanomateriales o productos que contengan nanomateriales, así como los enfoques de evaluación de la exposición; caracterización de los nanomateriales, desarrollo de normas y nomenclaturas uniformes, así como las técnicas de medición analítica; para los aspectos de salud ocupacional, la eficacia de una serie de medidas de gestión de riesgos, incluyendo confinamiento del proceso,

99 COMISIÓN EUROPEA, COMMUNICATION FROM THE COMMISSION TO THE EUROPEAN PARLAMENT, THE COUNCIL AND THE EUROPEAN ECONOMIC AND SOCIAL COMMITTEE, Regulatory aspects of nanomaterials, Bruselas, 2010, pp. 8-9. 
ventilación, equipo de protección personal, como equipos y guantes de protección respiratoria.

El desarrollo de normas y métodos de ensayo requiere la colaboración internacional para asegurar que los datos científicos pueden ser comparados y que los métodos científicos utilizados a efectos regulatorios están armonizados. Uno de los foros más destacables para la coordinación de actividades a nivel internacional ha sido organizado por el Grupo de Trabajo de la OCDE sobre Nanomateriales Manufacturados. El trabajo también se lleva a cabo en el marco de la Organización Internacional de Normalización, ISO.

En la actualidad, existe una amplia gama de actividades que se han iniciado al objeto de mejorar la base de conocimiento sobre la nanociencia (véase el documento de trabajo de la Comisión anexa y la Comunicación de la Comisión «Nanociencias y Nanotecnologías: Un plan de acción para Europa 2005-2009 Primer Informe de Ejecución 2005-2007)» ${ }^{100}$. Si bien, la labor de estos Grupos de trabajo, las reuniones de las autoridades competentes y organismos encargados debe ir más allá de coordinar la aplicación y los mecanismos de reglamentación. Así, tendrán que examinar de forma continua qué tipo de acción adicional se necesita. Estas actividades principalmente se reflejarán en los documentos que apoyan la implementación de la legislación vigente ${ }^{101}$.

Ejemplos de ello son el establecimiento de umbrales, la autorización de sustancias e ingredientes, la clasificación de residuos peligrosos, reforzando la evaluación de la conformidad por reclasificación, la introducción de restricciones a la comercialización y el uso de sustancias y preparados químicos, etc. Asimismo, se necesita trabajar en documentos de uso voluntario, como la orientación normativa, las normas europeas o internacionales, el asesoramiento de comités científicos, etc. Del mismo modo, las cuestiones éticas tienen que ser tratadas.

B. La necesidad de un consenso sobre el término «nanomaterial»

La falta de una regulación específica está generando problemas tan elementales como la simple adopción de conceptos básicos consensuados. Antes siquiera de iniciar un proceso regular es necesario acordar el contenido de determinados conceptos esenciales como es el de «nanomaterial».

La definición del término debe basarse en los conocimientos científicos disponibles. Para ello la medición del tamaño y la forma de distribución de los nanomateriales puede ser una posibilidad. 100 Vide http://ec.europa.eu/nanotechnology/pdf/comm_2007_0505_en.pdf.
101 Ídem. 
Al respecto, el Centro de Investigación de Referencia del informe de la Comisión Europea Conjunta sugirió que la definición de los nanomateriales debe abordar los nanomateriales de partículas, que sean ampliamente aplicable en la legislación de la Unión y estar en línea con otros enfoques en todo el mundo. El tamaño debe ser la propiedad que ayude a definir con mayor precisión la nanoescala.

La Comisión encargó al Comité Científico de Emergentes y Recientemente Identificados los Riesgos Sanitarios (CGRSERI) la tarea de proporcionar una aportación científica sobre los elementos a considerar en el desarrollo de una definición del término «nanomaterial» a efectos de regulación. La opinión «Base científica de la definición del término "nanomaterial"» fue objeto de una consulta pública en 2010. En su dictamen de 8 de diciembre de 2010, CGRSERI llegó a la conclusión de que el tamaño es universalmente aplicable a los nanomateriales y magnitudes de medición más adecuado. Un rango de tamaño definido facilitaría una interpretación uniforme. Se propuso el límite inferior a $1 \mathrm{~nm}$. Un límite superior de $100 \mathrm{~nm}$ es comúnmente utilizado por consenso general, pero no hay ninguna evidencia científica que apoye la idoneidad de este valor.

En 2011 la Unión Europea emitió una definición de nanomaterial, convirtiéndose en la única jurisdicción con una definición homogénea ${ }^{102}$. La definición queda establecida en la Recomendación «on the definition of nanomaterial» ${ }^{103}$.

Un nanomaterial como se define debe consistir en el 50\% o más de partículas que tienen un tamaño comprendido entre $1 \mathrm{~nm}$ y $100 \mathrm{~nm}^{104}$. Sin embargo, puede haber casos legislativos específicos donde las consecuencias para el medio ambiente, la salud, la seguridad o la competitividad justifiquen la aplicación de un umbral por debajo del $50 \%$.

102 FOLADORI, G. y INVERNIZZI, N., "La regulación de las nanotecnologías: una mirada desde las diferencias EUA-UE”, en Vigilancia Sanitaria Debate, 4, núm. 2 (2016), p. 12.

103 COMISIÓN EUROPEA, Recomendation of XXX on the definition of nanomaterial, Bruselas, 2011, p. 5: «"Nanomaterial" significa un material natural, accesorio o fabricado que contiene partículas, en un estado no unido o como un agregado o como un aglomerado y donde, para $50 \%$ o más de las partículas en la distribución de tamaño numérica, una o más dimensiones externas está en El intervalo de tamaños de $1 \mathrm{~nm}$ a $100 \mathrm{~nm}$. En casos específicos y cuando se justifiquen las preocupaciones por el medio ambiente, la salud, la seguridad o la competitividad, el umbral de distribución del tamaño numérico del $50 \%$ puede ser sustituido por un umbral entre el 1 y el $50 \%$ \%.

104 No hay ninguna base científica inequívoca que sugiera un valor específico para una distribución de tamaño distinto a $1 \mathrm{~nm}-100 \mathrm{~nm}$. Fuera de este rango, los materiales no pueden exhibir propiedades específicas de los nanomateriales. El asesoramiento científico era utilizar un enfoque estadístico basado en la desviación estándar con un valor umbral de 0,15\%. Dado el carácter generalizado de los materiales que serían cubiertos por dicho umbral y la necesidad de adaptar el alcance de la definición para su uso en un contexto normativo, el umbral debe ser mayor. 
El desarrollo tecnológico y los avances científicos continúan a gran velocidad. Por lo tanto, los criterios de definición deberán ser objeto de una revisión de forma periódica.

\section{La nanomedicina: medicamento vs producto sanitario. A propósito del Reglamento (UE) 2017/745}

Un importante reto que debía ser afrontado desde el ámbito jurídico de la UE era la reformulación de la desactualizada y dispersa regulación sobre productos sanitarios que quedaba regulada al amparo de la Directiva 90/385/CEE del Consejo, de 20 de junio de 1990, relativa a la aproximación de las legislaciones de los Estados Miembros sobre los productos sanitarios implantables activos, y la Directiva 93/42/ CEE del Consejo de 14 de junio de 1993 relativa a los productos sanitarios.

Este grupo normativo regulador, en vinculación con la Directiva 2001/83/CE del Parlamento Europeo y del Consejo de 6 de noviembre de 2001 por la que se establece un código comunitario sobre medicamentos para uso humano, diseña un marco de gran complejidad donde han surgido importantes conflictos como la difusa diferenciación entre medicamento y producto sanitario. Conviene recordar que la amplia aplicación de la nanomedicina ha derivado en el desarrollo tanto de medicamentos como de productos médicos, y en algunos casos se han combinado ambos. Este hecho y la falta de una norma reguladora representan una gran complejidad a la hora de identificar bajo qué categoría se sitúa el producto y, por lo tanto, qué regulación es aplicable.

Por ello, desde la UE se afrontó el desafío de establecer un nuevo punto de referencia jurídico mediante el desarrollo y aprobación del Reglamento (UE) 2017/745 del Parlamento Europeo y del Consejo de 5 de abril de 2017 sobre los productos sanitarios, por el que se modifican la Directiva 2001/83/CE, el Reglamento (CE) n. ${ }^{\circ}$ 178/2002 y el Reglamento (CE) n. ${ }^{\circ} 1223 / 2009$ y por el que se derogan las Directivas 90/385/CEE y 93/42/CEE del Consejo.

En virtud del art. 1.6.b) del Reglamento 2017/745, el mismo no será aplicable «a los medicamentos, tal como se definen en el artículo 1, punto 2, de la Directiva 2001/83/CE». Por consiguiente, debemos continuar acudiendo a la Directiva 2001/83/CE sobre medicamentos para uso humano, a la hora de identificar la definición de medicamentos (Medical Product):

«toda sustancia o combinación de sustancias que se presente como poseedora de propiedades curativas o preventivas con respecto a las enfermedades humanas; se considerarán asimismo medicamentos todas las sustancias o combinación de sustancias que puedan 
administrarse al hombre con el fin de establecer un diagnóstico médico o de restablecer, corregir o modificar las funciones fisiológicas del hombre» (artículo 1.2).

Por su parte, el reciente Reglamento (UE) 2017/745, en su art. 2.1) define los productos sanitarios (Medical Device) como:

«todo instrumento, dispositivo, equipo, programa informático, implante, reactivo, material $u$ otro artículo destinado por el fabricante a ser utilizado en personas, por separado o en combinación, con alguno de los siguientes fines médicos específicos: - diagnóstico, prevención, seguimiento, predicción, pronóstico, tratamiento o alivio de una enfermedad, - diagnóstico, seguimiento, tratamiento, alivio o compensación de una lesión o de una discapacidad, - investigación, sustitución o modificación de la anatomía o de un proceso o estado fisiológico o patológico, — obtención de información mediante el examen in vitro de muestras procedentes del cuerpo humano, incluyendo donaciones de órganos, sangre y tejidos, y que no ejerce su acción principal prevista en el interior o en la superficie del cuerpo humano por mecanismos farmacológicos, inmunológicos ni metabólicos, pero a cuya función puedan contribuir tales mecanismos. Los siguientes productos también se considerarán productos sanitarios: - los productos de control o apoyo a la concepción, - los productos destinados específicamente a la limpieza, desinfección o esterilización de los productos que se contemplan en el artículo 1, apartado 4, y en el párrafo primero del presente punto».

Así, podemos encontrarnos con 3 supuestos diferentes en el ámbito de la nanomedicina: un medicamento, que se regiría por la Directiva 2011/83/CE; un producto médico, el cual quedaría regulado por el Reglamento (UE) 2017/745; y finalmente, una aplicación que contuviera elementos de «medicamento» y de «producto médico».

En este último supuesto, el Reglamento (UE) 2017/745 viene a arrojar un poco de luz sobre el marco normativo aplicable. En su art. 1.7. indica que, cuando el producto incorpore como parte integrante una sustancia que, utilizada por separado, se consideraría un medicamento y que tenga una acción accesoria respecto a la del producto, deberá ser evaluado y autorizado con arreglo a lo establecido en dicho Reglamento. No obstante, si la acción de esa sustancia es principal y no es accesoria respecto a la del producto, el producto integrado estará regulado por la Directiva 2001/83/CE.

Sin embargo, el supuesto más complejo se presenta cuando el medicamento y el producto sanitario forman parte de un todo indivisible. En tal caso, el artículo 1.9. del Reglamento (UE) 2017/745 establece: «si el producto destinado a administrar un medicamento y el medicamento se introducen en el mercado de modo que constituyan un único producto integrado destinado a ser utilizado exclusivamente como tal y que no sea reutilizable, dicho producto único integrado estará regulado por la Directiva 2001/83/CE [...]. En ese caso, se aplicarán los 
requisitos generales de seguridad y funcionamiento pertinentes del anexo I del presente Reglamento en lo tocante a la seguridad y el funcionamiento de la parte que constituye un producto sanitario del producto único integrado».

En definitiva, si el producto es divisible será regulado por la Directiva 2001/83/ CE y el Reglamento (UE) 2017/745. No obstante, si el producto es indivisible la norma aplicable es la Directiva 2001/83/CE.

\section{La ética aplicada a la nanociencia}

La ciencia y tecnología actual que se desarrollan a nanoescala tienen un papel protagonista en la actividad científica actual. Las enormes expectativas que ha generado la nanociencia y la nanotecnología y las exigencias que rodean a este sector no pueden ser motivo suficiente para que su desarrollo no vaya acompañado por la supervisión adecuada y una regulación suficiente para erradicar o minimizar sus efectos negativos.

Aún nos situamos en una fase muy primitiva a tenor de las posibilidades que puede ofrecer la nanotecnología, y nos encontramos ante la posibilidad de, esta vez sí, hacer las cosas bien. La importancia que se desprende de la nanociencia la hace merecedora de un desarrollo sostenible, que le permita un paso firme, pero sin descuidar los riesgos que la rodean, que son numerosos e importantes. Es necesario dimensionar esos riesgos para controlarlos, pero sin olvidar el poder transformador de la nanotecnología. A tal fin, la ética puede ser clave para orientar y conformar la nanotecnología como bien colectivo de la sociedad ${ }^{105}$.

La complejidad de la nanotecnología como técnica interdisciplinaria, y su desarrollo aun primario hacen que establecer una regulación al respecto sea altamente complejo; como los juristas bien conocemos, el Derecho suele ir con retraso respecto de la sociedad. Es por esto que la aplicación de la ética a la nanociencia y la nanotecnología nos da la posibilidad de prever futuros problemas o por lo menos plantear cuestiones que permitan establecer un rumbo de desarrollo más adecuado. De este modo, la nanoética debe tener un carácter prospectivo, y anticiparse a la realización de los proyectos nanotecnológicos ${ }^{106}$, es decir, aplicar la ética a un futuro inexistente. Además, la ética ya ha sido aplicada al desarrollo de otras tecnologías por lo que su vinculación a la nanotecnología no debe ser muy diferente.

El acercamiento de la ética al mundo «nano» permite responder y valorar las cuestiones más relevantes vinculadas a este sector: ¿qué aplicaciones puede ofrecer

105 TOMÁS QUIRINO, C. y SANCÉN CONTRERAS, F. (2014: 58).

106 LINARES SALGADO, J. E., "Nanoética, un nuevo campo para viejos problemas del riesgo tecnológico", en Contrastes, Vol. XVIII (2013), p. 347. 
la nanotecnología y cuáles son sus efectos?, ¿cuáles pueden ser las consecuencias?, ¿se puede afrontar su desarrollo desde la equidad económica?, ¿se puede proteger el derecho a la salud cuando estamos expuestos a nanopartículas libres en el entorno?, ¿cómo se debe regular este sector?, etc.

Conviene resaltar que los problemas éticos que concibe la nanotecnología no son muy distintos de los que ha planteado el desarrollo de otras tecnologías.

Problemas como la distinción entre curación y mejoramiento, el acceso de toda la población a los nuevos tratamientos quirúrgicos, la manipulación de la información genética no sólo para la construcción de nuevos seres humanos, sino para la obtención de tejidos en el mismo organismo con propiedades diversas, traen necesariamente a discusión el problema de la ética para la práctica de la medicina. En este sentido, es necesario hacer referencia a la Ley 14/2007, de 3 de julio, de Investigación Biomédica, que tiene por objeto, a tenor de los rápidos avances, regular las pruebas genéticas y su implicación en los biobancos ${ }^{107}$.

Todo esto abre un campo de reflexión originado en la medicina, pero que toca aspectos universales del ser humano y que tradicionalmente se trata en el ámbito de la ética ${ }^{108}$. Es por esto que la relación de la medicina con la ética adquiere hoy día nuevos retos, derivada de la incorporación de la nanotecnología a la investigación y práctica médica.

Otra cuestión ética que debe ser planteada en torno a la nanotecnología (especialmente en su aplicación con la medicina) es la relacionada con la accesibilidad. La brecha social que existe actualmente provoca que una parte importante de la población tenga dificultades o le sea imposible acceder a las posibilidades que ofrecen avances tecnológicos como la nanotecnología. En este sentido, los Estados deben proteger la nanotecnología de una excesiva privatización que desemboque en altos costes y en opacidad.

Por otro lado, si como se espera de la nanotecnología se desarrollan nanomedicamentos más eficientes, es responsabilidad de las instituciones públicas garantizar un acceso igualitario a las mismos.

Esta opacidad es otra de las cuestiones importantes a tratar. El secretismo que caracteriza el desarrollo de la industria privada no puede generar en el desconocimiento de algunos de los inconvenientes que rodean a esta tecnología, todo ello bajo un ordenamiento jurídico ajeno a ella. Una manera de evaluar imparcialmente los riesgos y la adecuación tecnológica es fomentar la investigación científica inde-

107 MARTÍNEZ NAVARRO, J. A., "Genética y Derecho. La medicina genómica en el Sistema Nacional de Salud", en Revista Aragonesa de Administración Pública, núm. 54 (2019), pp. 337 y ss.

108 SANCÉN CONTRERAS, F. (2010: 77). 
pendiente de los intereses comerciales, bajo la supervisión de Estados u organismos internacionales ${ }^{109}$.

Nos encontramos ante una tecnología sin desarrollar plenamente, por lo que existe un claro vacío de conocimiento, más aún si tenemos en cuenta que casi la totalidad de los profesionales médicos carecen de cualidades para manejarla. A esto debemos añadir que las posibilidades de diagnóstico que ofrece esta tecnología pueden no ser del todo entendidas por los expertos.

Para concluir, expondré la idea que expresa Tomás Quirino y Sancén Contreras sobre la aportación de la ética al mundo nano: «Gracias a esta modificación de normas antes consideradas como absolutas, surge la necesidad de pensar una ética del devenir en la que más que ajustar la acción del hombre a normas consideradas como absolutas se asuma desde dicha acción la responsabilidad de la futura realidad que se construye a partir del actuar humano. Es desde esta perspectiva que consideramos a la nanociencia y los riesgos posibles que entraña su desarrollo y aplicación en bienes útiles para la sociedad. Así, dado el amplio espectro que cubren las nanociencias, la nanoética no consiste en denunciar el riesgo, sino que tiende a precisar su ubicación y dimensión para su posible manejo» ${ }^{110}$.

Por consiguiente, el objetivo de la ética debe ser favorecer y fomentar una investigación en el campo de la nanotecnología desde un enfoque positivo, es decir, en la búsqueda de aplicaciones cuya contribución sea responsable y sensible a los problemas sociales y alcance las expectativas ya fijadas, sin la intención de lastrar el desarrollo de este sector.

\section{MODO DE GONGLUSIÓN}

La nanotecnología ha llegado para rediseñar nuestra sociedad desde todos los ámbitos. Su desarrollo e influencia marcarán los principales avances científicos y tecnológicos de las futuras décadas.

109 MENDES DE BARROS, L., "Hibridación tecnológica, mediática y cultural. Las mediaciones culturales en el contexto de la globalización”, en Revista CIDOB d'Afers Internacionals, núm. 88 (2009), p. 149. Otra consecuencia de la aplicación de la nanotecnología, especialmente de la nanomedicina, cuya trascendencia ético-filosófica está siendo muy tratada es la denominada «hibridación tecnológica». La hibridación tecnológica surge cuando «en el mismo espacio social las manifestaciones artísticas populares y artesanías regionales se mezclan con los aparatos tecnológicos digitales del mundo globalizado». En este sentido, «[...] los signos de hibridación están en todas partes. El pasado y el presente se sobreponen y se articulan con las perspectivas del futuro. El tiempo físico diacónico encuentra su relatividad al proyectarse en otras escalas de tiempos prácticas, simbólicos». Recomiendo también: BALLESTAS, N. y RIVERA, P. E., "La hibridación tecnológica. Una aproximación al proceso comunicativo desde la perspectiva educativa”, en Panorama, núm. 7 (2009), pp. 21-27.

110 TOMÁS QUIRINO, C. y SANCÉN CONTRERAS, F. (2011: 59). 
Con carácter general, lo que se espera de la nanotecnología es la creación de nuevos materiales, dispositivos e instrumentos que no solamente introduzcan cambios, sino que supongan una auténtica revolución en áreas tan importantes como medicina, industria, seguridad, defensa, medioambiente, transporte, electrónica, educación, ocio, y un largo etcétera.

En su relación con el ámbito de la salud, la denominada «nanomedicina» está destinada a adoptar los nuevos mecanismos de diagnóstico y tratamiento que regirán la prestación sanitaria del futuro. Sus posibilidades se sitúan a la altura de la medicina genómica.

No obstante, todo avance tecnológico plantea retos. En efecto, la repercusión y el potencial de la nanotecnología hacen prever el desarrollo de importantes conflictos jurídicos. En particular, el Derecho tiene la obligación de establecer las reglas y pautas a partir de las cuales aplicar esta tecnología, acotando y afrontando todos los desafíos que presente.

Primeramente, será necesario diseñar un marco normativo regulador. El ordenamiento actual de la Unión Europea se estima como insuficiente cuanto menos para afrontar los retos jurídicos que se han planteado en campos como: la toxicidad de los nanomateriales, los efectos en el medioambiente y la salud pública, y, especialmente, en la patente de nuevos medicamentos diseñados a nanoescala.

Segundamente, es requisito indispensable establecer protocolos de desarrollo e investigación estandarizados. Para ello, será imprescindible acotar y consensuar términos esenciales a nivel internacional ${ }^{111}$.

Finalmente, el Derecho deberá ejercer como un medio de contención o conducción razonado que sea capaz de medir el impacto social de la ciencia y tecnología en este campo, para realizar los cambios de dirección que sean considerados como necesarios. Asimismo, la Administración Pública deberá velar por un uso no discriminatorio de todas las posibilidades que nos aportará el ámbito de la nanociencia.

111 RODRÍGUEZ MANZANERA, C., «Desde la filosofía del derecho, una mirada a la nanociencia y a la nanotecnología», en Revista de la Facultad de Derecho de la Universidad Nacional Autónoma de México, (2011), p. 249. 


\section{BIBLIOGRAFÍA}

AGUAYO GONZÁLEZ, F.; ZARZUELA ROLDÁN, E.; y LAMA RUIZ, J. R., "Nanotecnología y nanoquímica", en Sevilla Técnica, núm. 36 (2011).

ALGALÁ-ALGALÁ, S. y QUINTANAR-GUERRERO, D., "La terapia a nanoescala: ensamblaje de estructuras liberadoras de fármacos", en Revista Interdisciplinaria en Nanociencias y Nanotecnología, Vol. 7, núm. 12 (2014).

BALLESTAS, N. y RIVERA, P. E., "La hibridación tecnológica. Una aproximación al proceso comunicativo desde la perspectiva educativa", en Panorama, núm. 7 (2009).

BAWA, R., "FDA and Nanotech: Baby steps Lead to Regulatory Uncertainty, en MORIYAMA, H., y SHAHIDI, F., Bio-Nanotechnology: A revolution in Food, Biomedical and Health Sciences, Edita Debasis John Wiley \& Sons, Nueva York, 2013.

BECK, U., Risikogesellschaft. Auf dem Weg in eine andere Moderne, Frankfurt am Main, 1986.

BERTONE, P. y SNYDER, M., "Advances in functional protein microrray technology, en FEBS JOURNAL, 272, núm. 21 (2005).

GASTELL, P., "Nanotubos de carbono", en CORREIA, A. (Coord.), Nanociencia y Nanotecnología en España. Un análisis de la situación presente y de las perspectivas de futuro, Phantoms foundation, España, 2011.

CHAN, J. M.; VALENCIA, P. M.; ZHANG, L.; LANGER, R.; y FAROKHZAD, O. C., "Polymeric nanoparticles for drug delivery", en Methods in Molecular Biology, núm. 624 (2010).

COMISIÓN DE LAS COMUNIDADES EUROPEAS, COMUNICACIÓN DE LA COMISIÓN AL CONSEJO, AL PARLAMENTO EUROPEO Y AL COMITÉ ECONÓMICO Y SOCIAL EUROPEO, Nanociencias y nanotecnologias: Un plan de acción para Europa 2005/2009, Bruselas, 2005.

COMISIÓN DE LAS COMUNIDADES EUROPEAS, COMUNICACIÓN DE LA COMISIÓN AL PARLAMENTO EUROPEO, AL CONSEJO Y AL COMITÉ ECONÓMICO Y SOCIAL EUROPEO, Aspectos reglamentarios de los nanomateriales, Bruselas, 2008.

COMISIÓN DE LAS COMUNIDADES EUROPEAS, COMUNICACIÓN DE LA COMISIÓN, Hacia una estrategia europea para las nanotecnologías, Bruselas, 2004. 
COMISIÓN EUROPEA, Comunicación de la Comisión al Consejo, al Parlamento Europeo y al Comité Económico y Social Europea. Nanociencias y nanotecnologías: Un plan de acción para Europa 2005-2009, Bruselas, 2005.

COMISIÓN EUROPEA, Communication from the Commission to the European Parliament, the Council and the European Economic and Social Committee. Regulatory aspects of nanomaterials, Bruselas, 2008.

COMISIÓN EUROPEA, Recomendation of XXX on the definition of nanomaterial, Bruselas, 2011.

COMISIÓN EUROPEA, Nanotechnology: the invisible giant tackling Europe's future challenges, Publicaciones Oficina de la Unión Europea, Bélgica, 2013.

CORREIA, A.; PÉREZ, M.; SÁENZ, J. J.; y SERENA, P. A., "Nanoscience and nanotechnology: driving research and applications", en Physica Staus Solidi, Vol. 1 (2007).

CORREIA, A. y SERENA, P. A., "Introducción”, en A. CORRIEA (coord.), Nanociencia y Nanotecnología en España. Un análisis de la situación presente y de las perspectivas de futuro, Phantoms foundation, España, 2011.

CUADROS CELORRIO, M.; LLANOS MÉNDEZ, A.; y VILLEGAS PORTERO, R., Nanotecnología en Medicina. Informe de sintesis de tecnología emergente, Agencia de Evaluación de Tecnologías Sanitarias de Andalucía, Sevilla, 2010.

DE LA ROSA CRUZ, E., "Nanofotónica: luz + nanopartículas”, en Ide@s CONCYTEG, 24, núm. 2 (2007).

ECHANDI PACHECO, R., "Nanoelectrónica: la nueva era de la miniaturización y la física cuántica”, en Revista Rhombus, Vol. 2, núm. 5 (2006).

ECHEVERRÍA-CASTILLO, F., "Retos de este siglo: nanotecnología y salud”, en Revista Cubana Hematología, Inmunología y Hemoterapia, núm. 29 (2013).

ESTEVE PARDO, J., "Ciencia y Derecho ante los riesgos para la salud. Evaluación, decisión y gestión”, en Documentación Administrativa, núm. 265-266 (2003).

ESTEVE PARDO, J., "Convivir con el riesgo. La determinación del riesgo permitido”, en E. J. PÉREZ ALONSO, E. ARANA GARCÍA, J. L. SERRANO MORENO y P. MERGADO PACHECO, Derecho, globalización, riesgo y medio ambiente, Tirant lo Blanch, España, 2012.

ESTEVE PARDO, J., "Responsabilidad de la Administración y riesgos del desarrollo", en A. B. CASARES MARCOS y T. QUINTANA LÓPEZ (dirs.), La 
responsabilidad patrimonial de la Administración Pública: estudio general y ámbitos sectoriales, Vol. 2, Tirant lo Blanch, España, 2013.

ETPN, "The potential of nanomedicine: Why is small different", http://www. etp-nanomedicine.eu/public/about-nanomedicine/what-is-nanomedicine.

EUROPEAN CHEMICALS AGENGY, ECHA Services at a glance, 2014.

EUROPEAN CHEMICALS AGENGY, Programa de trabajo 2019. Aspectos destacados, 2019.

FERNÁNDEZ DE GATTA SÁNCHEZ, D. (Dir.) y otros, Grafeno e innovación tecnológica. Aspectos jurídicos, Editorial Ratio Legis, España, 2017.

FOLADORI, G., y INVERNIZZI, N., "La regulación de las nanotecnologías: una mirada desde las diferentes EUA-UE”, en Vigil. Sanit. Debate, 4, núm. 2 (2016).

GARCÍA MARTÍNEZ, J., "Nanotecnología: un sector estratégico en innovación y creación de valor", en Economía Exterior, núm. 44 (2008).

GARCÍA VIDAL, A., "Nuevo reglamento de la Unión Europea sobre los ensayos clínicos de medicamentos de uso humano", en Análisis Farmacéutico GAEP, (2014).

GONZÁLEZ, J. M.; LÓPEZ, M.; y RUÍZ, G., Informe de vigilancia tecnológica. Nanomedicina (vt 5), en DE LA SOTA RÍUS, J. (Coord.), CEIM y Dirección General de Universidades e Investigación, Madrid, 2006.

HARRIS, S., "The regulation of nanomedicine: Will the existing regulatory scheme of the FDA suffice?", en Richmond Fournal of Law \& Technology, Vol. 16, núm. 2 (2009).

IRACHE, J. M., "Nanomedicina: nanopartículas con aplicaciones médicas", en Anales del Sistema Sanitaro en Navarra., Vol. 31, núm. 1 (2008).

JIMÉNEZ SIMÓN, N., "Productos cosméticos: marco normativo y prevención de riesgos laborales", en Notas Técnicas de Prevención (NTP), núm. 1074 (2016).

LECHUGA, L. M., "Nanomedicina: ampliación de la nanotecnología en la salud", Curso de Biotecnología aplicada a la salud humana, Grupo de nanobiosensores y aplicaciones bioanalíticas, Consejo Superior de Investigaciones Científicas, Madrid, 2010.

LINARES SALGADO, J. E., "Nanoética, un nuevo campo para viejos problemas del riesgo tecnológico", en Contrastes, Vol. XVIII (2013). 
LOERA SERNA, S.; RUIZ ÁNGELES, J.; FLORES MORENO, J.; y SOTO PORTAS, L.; "Protegiendo fármacos con nanomateriales inteligentes", en Revista Interdisciplinaria en Nanociencia y Nanotecnología, Vol. 5, núm. 1 (2012).

LOZANO, A., "El nanomagnetismo y la nanóptica centrarán parte de la labor de nanoGUNE”, en Diario Tecnología Vasca, 11 de junio de 2011.

MARTÍNEZ NAVARRO, J. A., "Genética y Derecho. La medicina genómica en el Sistema Nacional de Salud”, en Revista Aragonesa de Administración Pública, núm. 54 (2019).

MEJIAS SÁNCHEZ, Y.; CABRERA CRUZ, N.; TOLEDO FERNÁNDEZ, A. M.; y DUANY MACHADO, O. J., "La nanotecnología y sus posibilidades de aplicación en el campo científico-tecnológico", en Revista Cubana de Salud Pública, Vol. 35, núm. 3 (2009).

MENDES DE BARROS, L., "Hibridación tecnológica, mediática y cultural. Las mediaciones culturales en el contexto de la globalización", en Revista CIDOB d’Afers Internacionals, núm. 88 (2009).

MIRANDA SORIANO, R., "El tsunami de la nanotecnología", resumen ejecutivo de la sesión impartida el 11 de marzo, Madrid, 2015.

MONTANO, P. J., "La responsabilidad de médicos y científicos ante las nuevas tecnologías biogenéticas", en Cuadernos de Bioética, núm. 1 (1995).

MORENO GONZÁLEZ, J. y VALENZUELA JUAN, R., "Nanotecnología aplicada a la salud", en Revista I+D, núm. 79 (2010).

MORENO MOLINA, A. M., "Productos químicos. Biotecnología. Acuerdos Voluntarios", en Juan PICÓN RISQUEZ (Coord.), Derecho medioambiental de la Unión Europea, McGraw-Hill Interamericana de España, España, 1996.

MUÑOZ PÉREZ, D. y BERNAL LLUCH, R. Ma.., "Análisis del impacto de la nanomedicina: consideraciones jurídicas", en Aletheia, Cuadernos Críticos del Derecho, núm. 2 (2018).

Organización de Estados Iberoamericanos, La nanotecnología en Iberoamérica. Situación actual y tendencias, 2009, http://www.oei.es/salactsi/nano.pdf.

ODDONE, N.; ZAMBRANA, A.; BERVEJILlO, V.; ALBERRO, A.; y otros, "De la nanobiomoléculas a la nanobiología y nanomedicina", en Revista Interdisciplinaria en Nanociencias y Nanotecnología, Vol. 6, núm. 10 (2013). 
PÉREZ GÁlVEZ, J. F., "La sostenibilidad del Sistema Nacional de Salud", en J. F. PÉREZ GÁLVEZ (dir.), La sostenibilidad del Sistema Nacional de Salud en el siglo XXI, Comares, Granada, 2015.

POOLE, G. P. y OWENS, F. J., "Introduction to the Nanotechnology", en Wiley-VCH, Weinheim, (2003).

PRIETO E., "Nanometrología", en A. CORREIA (coord.), Nanociencia y Nanotecnología en España. Un análisis de la situación presente y de las perspectivas de futuro, Phantoms foundation, España, 2011.

ROCO, M. y BRAINBRIDGE, W. S., "Societal Implications of Nanoscience and Nanotechnology", Kluwer Press, (2001), http://www.wtec.org/loyola/nano/ NSET.Societal.Implications.

RODRÍGUEZ MANZANERA, C., "Desde la filosofía del derecho, una mirada a la nanociencia y a la nanotecnología", en Revista de la Facultad de Derecho de la Universidad Nacional Autónoma de México, (2011).

SAMITIER, J., "Nanobiología y Nanomedicina", en A. CORREIA (coord.), Nanociencia y Nanotecnología en España. Un análisis de la situación presente y de las perspectivas de futuro, Phantoms foundation, España, 2011.

SAMITIER, J., "Nanomedicina: nanotecnologías aplicadas a la salud", en Dossier Cientifico SEBBM, núm. 168 (2011).

SANCÉN GONTRERAS, F., "Nanoética y nanomedicina. Apuntes para una nueva ética de la medicina", en Revista Interdisciplinaria en Nanociencia y Nanotecnología, Vol. 3, núm. 1 (2010).

SANDERSON, K., "Putting nanotechnology regulation under the microscope", en The Guardian, 24 de junio de 2013.

SANZ CEBALLOS, L.; FERRIT MARTÍN, M.; y otros, "Novedades introducidas por el nuevo Real Decreto legislativo 1090/2015 de ensayos clínicos con medicamentos en España”, en Ars Pharm., 57, núm. 4 (2016).

SERENA, P. A.; y CORREIA, A., "Nanotecnología: el motor de la próxima revolución tecnológica", en Apuntes de Ciencia y Tecnología, núm. 9 (2003).

SERENA, P. A.; GORREIA, A.; SÁENZ, J. J.; y PÉREZ, M., "Nanotechnology applications: a driving force for R\&D investment", en Physica Staus Solidi, Vol. 204 (2007). 
SERENA, P. A., y TUTOR, J. D., "La divulgación y la formación de la nanociencia y la nanotecnología en España: un largo camino por delante", en Revista Interdisciplinar de Nanociencia y Nanotecnología, Vol. 4, núm. 2 (2011).

TOMA-BIANOV, A., "European Union Regulations on nanomedicine", en Bulletin of the Transilvania University of Brasov, Vol. 5 (54), núm. 2 (2012).

TOMÁS QUIRINO, C. y SANCÉN CONTRERAS, F., "Ubicación de riesgos en el trabajo a nanoescala. Una visión desde la ética", en Revista Interdisciplinar de Nanociencia y Nanotecnología, Vol. 4, núm. 1 (2011).

VIDAL CORREA, L., y ZAYAGO LAU, E., "Nanomedicina: expectativas y regulación para el desarrollo”, en Observatorio del desarrollo, Vol. 2, núm. 8 (2014).

ZEA, R., "Nanomaterials: health effects and legislation", en Ingeniería e investigación, Vol. 32, núm. 1 (2012). 